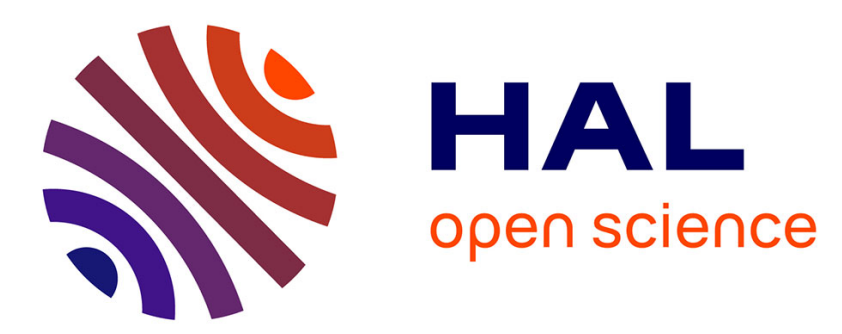

\title{
Involvement of Arabidopsis glutaredoxin S14 in the maintenance of chlorophyll content
}

\author{
Pascal Rey, Noëlle Becuwe, Sébastien Tourrette, Nicolas Rouhier
}

\section{To cite this version:}

Pascal Rey, Noëlle Becuwe, Sébastien Tourrette, Nicolas Rouhier. Involvement of Arabidopsis glutaredoxin S14 in the maintenance of chlorophyll content. Plant, Cell and Environment, 2017, 40 (10), pp.2319-2332. 10.1111/pce.13036 . hal-01686869

\section{HAL Id: hal-01686869 \\ https://hal.science/hal-01686869}

Submitted on 17 May 2018

HAL is a multi-disciplinary open access archive for the deposit and dissemination of scientific research documents, whether they are published or not. The documents may come from teaching and research institutions in France or abroad, or from public or private research centers.
L'archive ouverte pluridisciplinaire HAL, est destinée au dépôt et à la diffusion de documents scientifiques de niveau recherche, publiés ou non, émanant des établissements d'enseignement et de recherche français ou étrangers, des laboratoires publics ou privés. 


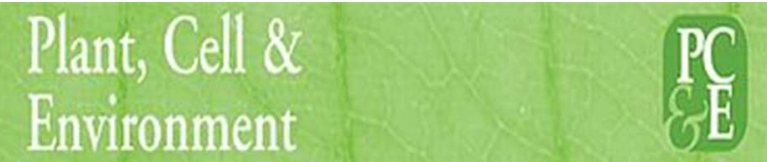

\section{Involvement of Arabidopsis glutaredoxin S14 in the maintenance of chlorophyll content}

\begin{tabular}{|c|c|}
\hline Journal: & Plant, Cell \& Environment \\
\hline Manuscript ID & Draft \\
\hline Wiley - Manuscript type: & Original Article \\
\hline Date Submitted by the Author: & $\mathrm{n} / \mathrm{a}$ \\
\hline Complete List of Authors: & $\begin{array}{l}\text { Rey, Pascal; CEA, UMR } 7265 \\
\text { Bécuwe, Noëlle; CEA, UMR } 7265 \\
\text { Tourrette, Sébastien; CEA, UMR } 7265 \\
\text { Rouhier, Nicolas; Université de Lorraine, UMR Interactions Arbres- } \\
\text { Microorganismes }\end{array}$ \\
\hline Environment Keywords: & salinity, oxidative stress \\
\hline Physiology Keywords: & growth, photosynthesis: electron transport \\
\hline Other Keywords: & chlorophyll, glutaredoxin, redox, senescence \\
\hline Abstract: & $\begin{array}{l}\text { Plant class-II glutaredoxins (GRXs) are oxidoreductases carrying a CGFS } \\
\text { active site signature and are able to bind iron-sulfur clusters in vitro. In } \\
\text { order to explore the physiological functions of the two plastidial class-II } \\
\text { isoforms, GRXS14 and GRXS16, we generated knockdown and } \\
\text { overexpression Arabidopsis thaliana lines and characterized their } \\
\text { phenotypes using physiological and biochemical approaches. Plants } \\
\text { deficient in one GRX did not display any growth defect, whereas the growth } \\
\text { of plants lacking both was slowed. Plants overexpressing GRXS14 exhibited } \\
\text { reduced chlorophyll content in control, high light and high salt conditions. } \\
\text { However, when exposed to prolonged darkness, plants lacking GRXS14 } \\
\text { showed accelerated chlorophyll loss compared to WT and overexpression } \\
\text { lines. We observed that the GRXS14 abundance and the proportion of } \\
\text { reduced form were modified in WT upon darkness and high salt. The dark } \\
\text { treatment also resulted in decreased abundance of proteins involved in the } \\
\text { maturation of iron-sulfur proteins. We propose that the phenotype of } \\
\text { GRXS14-modified lines results from its participation in the control of } \\
\text { chlorophyll content in relation with light and osmotic conditions, possibly } \\
\text { through a dual action e.g. regulating the redox status of biosynthetic } \\
\text { enzymes and contributing to the biogenesis of iron-sulfur clusters, which } \\
\text { are essential cofactors in chlorophyll metabolism. }\end{array}$ \\
\hline
\end{tabular}




\author{
SCHOLARONE ${ }^{\text {m }}$ \\ Manuscripts
} \\ Page 1 of $53 \quad$ Plant, Cell \& Environment
}

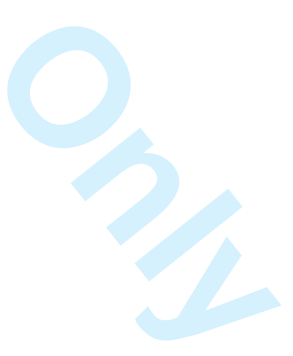


1 Involvement of Arabidopsis glutaredoxin S14 in the maintenance of chlorophyll content

2

3

4 Pascal Rey ${ }^{1,2,3^{*}}$, Noëlle Becuwe ${ }^{1,2,3}$, Sébastien Tourrette ${ }^{1,2,3}$, Nicolas Rouhier ${ }^{4,5}$

$5{ }^{1}$ CEA, DRF, BIAM, Lab Ecophysiol Molecul Plantes, Saint-Paul-lez-Durance, F-13108,

6 France

$7{ }^{2}$ CNRS, UMR 7265 Biol Veget \& Microbiol Environ, Saint-Paul-lez-Durance, F-13108,

8 France

$9 \quad{ }^{3}$ Aix-Marseille Université, Saint-Paul-lez-Durance, F-13108, France

$10{ }^{4}$ Université de Lorraine, Interactions Arbres - Microorganismes, UMR1136, F-54500

11 Vandoeuvre-lès-Nancy, France

$12{ }^{5}$ INRA, Interactions Arbres - Microorganismes, UMR1136, F-54280 Champenoux, France

13

14 * Corresponding author: Pascal Rey

15 CEA, DRF, BIAM, Laboratoire d'Ecophysiologie Moléculaire des Plantes,

16 Bâtiment 158

17 CEA-Cadarache

18

19

20

21

22

23

24

25

Short running title: Control of chlorophyll content by glutaredoxins 


\section{Abstract}

Plant class-II glutaredoxins (GRXs) are oxidoreductases carrying a CGFS active site signature and are able to bind iron-sulfur clusters in vitro. In order to explore the physiological functions of the two plastidial class-II isoforms, GRXS14 and GRXS16, we generated knockdown and overexpression Arabidopsis thaliana lines and characterized their phenotypes using physiological and biochemical approaches. Plants deficient in one GRX did not display any growth defect, whereas the growth of plants lacking both was slowed. Plants overexpressing GRXS14 exhibited reduced chlorophyll content in control, high light and high salt conditions. However, when exposed to prolonged darkness, plants lacking GRXS14 showed accelerated chlorophyll loss compared to WT and overexpression lines. We observed that the GRXS14 abundance and the proportion of reduced form were modified in WT upon darkness and high salt. The dark treatment also resulted in decreased abundance of proteins involved in the maturation of iron-sulfur proteins. We propose that the phenotype of GRXS14-modified lines results from its participation in the control of chlorophyll content in relation with light and osmotic conditions, possibly through a dual action e.g. regulating the redox status of biosynthetic enzymes and contributing to the biogenesis of iron-sulfur clusters, which are essential cofactors in chlorophyll metabolism.

Keywords: Arabidopsis thaliana, chloroplast, chlorophyll, glutaredoxin, iron-sulfur cluster, redox, senescence. 


\section{Introduction}

Glutaredoxins (GRXs) are small and ubiquitous oxidoreductases belonging to the thioredoxin (TRX) superfamily (Rouhier et al. 2008; Meyer et al. 2009). They display a 4-residue activesite signature including one or two redox-active cysteines that allow the reduction of disulfide bonds usually using glutathione (GSH) as an electron donor (Rouhier et al. 2006). Compared to other organisms, higher plants are characterized by an elevated number of $G R X$ genes, at least 31 in Arabidopsis thaliana, which are subdivided into four classes (Couturier et al. 2009). Class-I and -II GRXs exhibit in most cases CPXC and CGFS active site motifs, respectively, and are present in all photosynthetic organisms. GRXs from class III display a CCXX active site sequence, are restricted to terrestrial plants and are the most represented with 21 isoforms in A. thaliana. The last class (IV) is found in algae and terrestrial plants and contains proteins with one N-terminal GRX domain carrying a CXXC/S motif and two other domains of unknown function. Interestingly, several plant class-I and -II GRXs bind ironsulfur (Fe-S) clusters in vitro (Rouhier et al. 2007; Bandyopadhyay et al. 2008; Rouhier et al. 2010). Consistently, the yeast mitochondrial class-II GRX5 participates in Fe-S cluster assembly by transferring preformed Fe-S clusters to other specific carrier proteins before final insertion into acceptor proteins (Rodriguez-Manzaneque et al. 2002). In other respects, the Fe$\mathrm{S}$ cluster in human class-I GRX2, which is dissociated in the presence of oxidized glutathione, might play a redox sensor role by restoring the reductase activity that is lost upon Fe-S cluster ligation (Lillig et al. 2005). The participation of class-II GRXs in Fe-S cluster assembly in vivo in plants remained elusive until recent reports on Arabidopsis lines expressing mitochondrial GRXS15 variant forms which displayed decreased activity of aconitase, an FeS cluster-containing enzyme (Moseler et al. 2015), and on knockdown lines showing defects in the maturation of the mitochondrial lipoate synthase, an Fe-S enzyme (Ströher et al. 2016). Concerning the nucleo-cytosolic GRXS17, the evidence comes from its association with 
known cytosolic Fe-S cluster assembly components of the cytosolic machinery and with several putative Fe-S client proteins (Inigo et al. 2016).

In the last years, plant GRXs have been shown to fulfil key roles in developmental processes. For instance, in Arabidopsis, the deficiency in both class-I GRXC1 and GRXC2 results in a lethal phenotype due to impaired embryo development (Riondet et al. 2012). Two class-III GRXs participate in the development of floral organs via interaction with bZIP-type TGA transcription factors (Xing et al. 2005; Xing and Zachgo 2008). Another evidence of the involvement of class-III GRXs in plant reproduction is illustrated by the rice male sterile mill mutant (Hong et al. 2012). OsMIL1 encodes a GRX involved in the meiotic entry of sporogenous cells. Consistently, the fate of germ cells in maize anthers depends on a class-III GRX, which also regulates meristem functioning, presumably via interaction with a TGA transcription factor (Chaubal et al. 2003; Kelliher and Walbot, 2012; Yang et al. 2015). The class-II GRXS17, which is composed of one TRX-like domain and three GRX domains containing each a CGFS motif, plays a key role in the control of plant development in relation with environment. Arabidopsis grxs 17 plants grown at $28^{\circ} \mathrm{C}$ exhibit reduced primary root growth and impaired flowering (Cheng et al. 2011) and this mutant also displays a strongly impaired vegetative and reproductive development under long-day conditions (Knuesting et al. 2015). GRXS17, which interacts with the NF-YC11/NC2 $\alpha$ transcription factor, has been proposed to relay a redox signal generated by environmental conditions and required for proper apical meristem functioning. It is worth mentioning that the roles of class-II GRXs in plant development could be fulfilled through interplays with signalling pathways involving hormones like auxin (Cheng et al. 2011).

Besides their functions in development, plant class-III GRXs also participate in responses to environmental constraints such as photooxidative stress (Laporte et al. 2012) and to pathogens (Ndamukong et al. 2007; La Camera et al. 2011). With regard to class-II GRXs, 
Arabidopsis plastidial GRXS14 and mitochondrial GRXS15, which consist of a unique GRX domain, are presumed to play important roles upon oxidative stress, high temperature and arsenic exposure (Cheng et al. 2006; Sundaram et al. 2009; Sundaram and Rathinasabapathi, 2010; Ströher et al. 2016). Regarding the other plastidial class-II GRX, Guo et al. (2010) reported that tomato plants silenced for GRXS16 expression are more sensitive to osmotic constraints. Of note, GRXS16 is composed of two modules, an N-terminal domain which possesses endonuclease activity fused to a C-terminal GRX domain (Liu et al. 2013). This could suggest a link between redox regulation and plastidial DNA metabolism.

In the present work, we undertook a genetic approach to investigate the physiological functions of the two plastidial class-II GRXs in the view of their dual in vitro biochemical functions (reductase activity vs Fe-S cluster ligation). Our data revealed that GRXS14 and GRXS16 play important roles in the control of vegetative growth, and that GRXS14 possesses specific functions in the maintenance of chlorophyll content depending on environmental and light conditions.

\section{Materials and methods}

\section{Plant material, growth conditions and environmental constraints}

In standard conditions, Arabidopsis thaliana Col-0 plants were grown in soil in phytotron under an 8-h photoperiod and a photon flux density of $200 \mu \mathrm{mol}$ photons $\mathrm{m}^{-2} \cdot \mathrm{s}^{-1}$ at $22^{\circ} \mathrm{C}$. Several environmental constraints were applied on soil-grown plants (Cerveau et al. 2016). A gradually increasing water deficit was applied on 5-week-old plants by withholding watering for $c a 7$ days. For high light and high temperature treatment, 4-week-old plants were transferred at $825 \mu \mathrm{mol}$ photons $\mathrm{m}^{-2} \cdot \mathrm{s}^{-1}$ under an 8 -h photoperiod and $30^{\circ} \mathrm{C} / 18^{\circ} \mathrm{C}$ (day/night) for 1 week. Another high light condition was applied from sowing at $450 \mu \mathrm{mol}$ photons $\mathrm{m}^{-2} . \mathrm{s}^{-}$ ${ }^{1}$ and $22^{\circ} \mathrm{C} / 18^{\circ} \mathrm{C}$ (day/night, 16-h photoperiod) for 3 weeks. Four-week old plants were 
124

125

126

127

128

129

130

exposed to high salinity by watering with a solution of $150 \mathrm{mM} \mathrm{NaCl}$ for either 2 weeks or 1 month in the standard phytotron light conditions. For low temperature treatment, 3-week-old plants were transferred at $10^{\circ} \mathrm{C}$ in a growth chamber $\left(200 \mu \mathrm{mol}\right.$ photons $\mathrm{m}^{-2} \cdot \mathrm{s}^{-1}, 8-\mathrm{h}$ photoperiod) for 4 weeks. For photooxidative conditions, 5-week-old plants were exposed in a growth chamber to $1400 \mu \mathrm{mol}$ photons $\mathrm{m}^{-2} \cdot \mathrm{s}^{-1}$ (8-h photoperiod) at $6^{\circ} \mathrm{C}$ for 7 days. For senescence experiments, well-expanded leaves of 6-week-old plants were covered with aluminum foil and plants were further cultivated in the phytotron in standard conditions for 8 days before analysis of covered leaves.

Osmotic and oxidative constraints were also investigated on leaf disks by performing incubation on water, $150 \mathrm{mM} \mathrm{NaCl}, 150 \mathrm{mM} \mathrm{KCl}, 300 \mathrm{mM}$ mannitol or $2 \mu \mathrm{M}$ methyl viologen with the leaf abaxial side directly in contact with the solution. Chemicals were purchased from Sigma. Discs (1.3-cm diameter) were excised from adult well-expanded leaves from 6- to 7-week old Arabidopsis plants grown in control conditions and floated for $48 \mathrm{~h}$ in the phytotron conditions $\left(200 \mu \mathrm{mol}\right.$ photons. $\mathrm{m}^{-2} \cdot \mathrm{s}^{-1} ; 8$-h photoperiod $)$ or in continuous dark for 2 to 5 days.

\section{Transformation of $\boldsymbol{A}$. thaliana plants and PCR analyses}

The full-length GRXS14 and GRXS16 coding sequences were cloned into the pB2GW7 or pH2GW7 vectors (GATEWAY® Invitrogen) for overexpression and the partial GRXS16 coding sequence into the pK7GWIWG2(I) vector (GATEWAY ${ }^{\circledR}$ Invitrogen) for RNA interference using appropriate primers (Table S1). Following transformation using Agrobacterium tumefaciens C58 strain (Clough and Bent, 1998), homozygous lines (T2) were obtained from resistance segregation assays. Leaf genomic DNA was extracted using the DNeasy Plant Mini Kit (Qiagen) to perform PCR using appropriate primers (Table S1), Taq 
DNA polymerase (Life Technologies) and the GeneAmp PCRSystem 2700 (Applied Biosystems).

150

\section{Protein preparation, electrophoresis and Western blot analysis}

Organs from Arabidopsis were ground in liquid nitrogen and the powder was suspended in 50 $\mathrm{mM}$ Tris- $\mathrm{HCl} \mathrm{pH} 8,1 \mathrm{mM}$ PMSF and $50 \mathrm{mM} \beta$-mercaptoethanol to prepare soluble proteins as described in Rey et al. (2005). For extraction in non-reducing conditions, $\beta$ mercaptoethanol was omitted. Following vigorous shaking at $4^{\circ} \mathrm{C}$ for $20 \mathrm{~min}$ and centrifugation (20 min, 15,000 $\mathrm{rpm}, 4^{\circ} \mathrm{C}$ ), the supernatant was precipitated by adding 2 volumes of acetone. Protein concentration was determined using the "Protein Quantification BCA Assay" kit (Interchim, France). Proteins were separated using SDS-PAGE and electroblotted onto $0.45 \mu \mathrm{m}$ nitrocellulose membranes (Pall Corporation, NY, USA) to carry out immunoblot analysis (Rey et al. 2005). The primary antibodies used were raised in rabbit (Table S2) and detected using the goat anti-rabbit “Alexa Fluor ${ }^{\circledR} 680 " \operatorname{IgG}(1: 10,000)$ (Invitrogen, Carlsbad, USA). Revelation and quantification of band intensity were performed using the Odyssey Infrared Imager (Licor, Lincoln, NE, USA).

164

\section{mPEG maleimide labelling}

Leaf soluble proteins were prepared as described above in $50 \mathrm{mM}$ Tris- $\mathrm{HCl} \mathrm{pH} 8,1 \mathrm{mM}$

PMSF in the absence of reductant. Proteins were precipitated using 2 volumes of acetone at $20^{\circ} \mathrm{C}$ for $2 \mathrm{~h}$ and immediately used for labelling. Following centrifugation, an aliquot of 200

$169 \mu \mathrm{g}$ protein was suspended in PBS, $1 \%$ SDS and $1.7 \mathrm{mg} \cdot \mathrm{mL}^{-1} \mathrm{mPEG}-$ maleimide-2000 (Laysan Bio Arab, AL, USA) and incubated at room temperature for $3 \mathrm{~h}$. Finally, the reaction mixture and Western blot analyses as described above. 


\section{Chlorophyll content determination}

175 One-cm diameter leaf disks were taken from well-expanded adult leaves, stored at $-80^{\circ} \mathrm{C}$ and 176 crushed in $1 \mathrm{~mL} 80 \%$ acetone. Following vigorous shaking, overnight storage in the dark at $4^{\circ} \mathrm{C}$ and centrifugation $(14,000 \mathrm{~g}, 10 \mathrm{~min})$, the content in chlorophylls $a$ and $b$ was measured spectrophotometrically and calculated according to Lichtenthaler (1987).

\section{Chlorophyll fluorescence measurements}

Chlorophyll fluorescence emission from the upper leaf surface was measured at room temperature using a PAM-2000 modulated Walz fluorometer (Effeltrich, Germany; Havaux et al. 2000). After dark adaptation for $20 \mathrm{~min}$, the initial level $\left(\mathrm{F}_{0}\right)$ of chlorophyll fluorescence was excited by a dim red light modulated at $600 \mathrm{~Hz}$ and determined after a $1 \mathrm{~s}$ illumination with far-red light. The maximal level of chlorophyll fluorescence $\left(\mathrm{F}_{\mathrm{m}}\right)$ was induced by a pulse of intense white light. The maximal quantum yield of photosystem II (PSII) photochemistry was calculated as $\left(\mathrm{F}_{\mathrm{m}}-\mathrm{F}_{0}\right) / \mathrm{F}_{\mathrm{m}}=\mathrm{F}_{\mathrm{v}} / \mathrm{F}_{\mathrm{m}}\left(\mathrm{F}_{\mathrm{v}}\right.$ : variable fluorescence). PSI and PSII chlorophyll fluorescence emission spectra at low temperature were measured from leaf discs in liquid nitrogen using a PerkinElmer LS50B luminometer equipped with fiber optic light guides (Havaux et al. 2000). 


\section{Results}

\section{Expression of GRXS14 and GRXS16 during development and environmental} constraints in Arabidopsis

The information about the plastidial GRXS14 and GRXS16 levels being poor, we first investigated their abundance using Western blot analysis. Soluble protein extracts were prepared from leaves at various developmental stages (young less than one $1 \mathrm{~cm}$ long, wellexpanded and old non-senescent) of WT Arabidopsis plants grown in short-day conditions for six weeks (rosette stage) or from various organs of plants cultivated in long-day conditions for the same period (reproductive stage). Fig. 1a shows that GRXs have quite similar distribution patterns and are present in most organs. GRXS14 was found in all organs, although barely detectable in seeds, and displayed a higher abundance in photosynthetic organs particularly young leaves, as compared to roots, flower buds and flowers. In comparison, the GRXS16 protein was slightly detected in root extracts and its abundance was almost as high in buds and flowers as in leaves and stems.

Then we analyzed the abundance of GRXs in leaves of soil-grown plants exposed to high light in long day (HL-LD), high light and high temperature (HL-HT), photooxidative treatment (PT), low temperature (LT), high salinity (S) and water deficit (WD). Upon these constraints, no substantial change was observed in the abundance of RubisCO and 2-Cys peroxiredoxin (PRX) (Cerveau et al. 2016). Decreased GRXS14 protein levels were observed in high light conditions (HL-LD and HL-HT), whereas strongly increased amounts were noticed upon osmotic constraints, i.e. low temperature, salt and drought $(+c a 100,150$ and $200 \%$, respectively, Fig. 1b). In contrast, no change was observed regarding the GRXS16 abundance except upon high light conditions (HL-LD and HL-HT), which led to protein levels reduced by ca $30 \%$ (Fig. 1b). These data reveal differential abundance of the two plastidial class-II GRXs in response to environmental constraints. 
Generation of Arabidopsis lines modified in the expression of GRXS14 and/or GRXS16

Homozygous Arabidopsis plants knockout for GRXS14 expression at the protein level were selected from the SALK_125902 line termed K. Two independent lines, termed O1 and O2

227 (Table 1), overexpressing GRXS14 in the WT background were selected based on a much higher protein level in leaf extracts compared to WT (Fig. 2a,c). Note that in these 3 lines, the GRXS16 abundance was not altered. In parallel, we transformed WT plants with the GRXS16 cDNA under the control of the CaMV-35S promoter. Two types of lines, either overexpressing GRXS16 at a high level or lacking the protein, very likely due to a cosuppression phenomenon, were obtained (Table 1, Fig. S1a, b). In the 4 lines either up- or down-regulated for GRXS16 expression selected for further studies, no change in leaf GRXS14 abundance was observed (Fig. S1b). Finally, we generated Arabidopsis plants down-regulated for the expression of both GRX genes. Due to the lack of T-DNA insertion mutants for GRXS16 and the instability of co-suppression mechanisms in the progeny, we developed a RNA-interference strategy in the grxS14 genetic background. Two lines termed KI1 and KI2 (Table 1) displaying a leaf GRXS16 abundance decreased by ca 50 and $80 \%$, respectively, compared to WT and grxS14 (K) plants, were selected (Fig. 2b,d).

\section{Characterization of plants modified for GRX14 and GRXS16 expression}

We first compared the growth of modified lines by measuring the rosette weight of 6-weekold plants in control conditions. We noticed that germination was similar for all lines. Compared to WT, we observed no difference regarding lines knockout for GRXS14 expression (K), down-regulated for GRXS16 expression (C2 and $\mathrm{C} 4)$ or overexpressing this gene (O4 and O5) (Fig. 3a). In contrast, at the same age, the rosette weight of lines overexpressing GRXS14 (O1 and O2) was significantly increased by ca 20\% (2.75 g) 
compared to that of WT $(2.3 \mathrm{~g})$ whereas grxS14 lines knockdown for GRXS16 expression (KI1 and KI2) displayed a substantially reduced rosette weight (ca -25\%, 1.75 g) (Fig. 3a, b). Of note, the growth of the 2 lines restored for GRXS14 expression (KI1-R1 and KI1-R2, Table 1) was similar to that of WT (Fig. S2a, b). We then focused our study on the lines modified for GRXS14 expression (K, O1 and $\mathrm{O} 2$ ) and those knockout for GRXS14 and down-regulated for GRXS16 (KI1 and KI2) to investigate some photosynthetic parameters. In control conditions, no difference in the maximal PSII photochemical efficiency, assessed by the $F_{v} / F_{m}$ parameter, was noticed (Fig. S3a). In terms of leaf chlorophyll content, both lines overexpressing GRXS14 displayed a significantly lower level compared to that in WT and no variation was noticed in other lines (Fig. 3c). Finally, as plastidial GRXS14 and S16 are presumed to participate in the maturation of $\mathrm{Fe}-\mathrm{S}$ proteins, we examined parameters related to two major photosynthetic actors containing Fe-S clusters, i.e. photosystem I and ferredoxin (FDX). Low temperature fluorescence emission spectra measured in leaf disks, which reflect the organization of PSI and PSII complexes, and Western analysis of FDX2 abundance did not reveal any difference in GRX-modified lines (Fig. S4a, b). Looking at the content of some actors involved in the maturation of plastidial Fe-S proteins, we observed that the abundance of NFU2 and SUFE1 was decreased by $c$ a $30 \%$ in lines overexpressing GRXS14 or GRXS16, whereas no change was noticed for other proteins (NFU3, NFS2, SUFA1, SUFB) (Fig. S4b, c, d). Collectively, these results indicate that plants modified in the expression of plastidial class-II GRXs exhibit a growth phenotype associated with alterations in the chlorophyll content and in the abundance of some actors involved in the maturation of Fe-S proteins.

\section{Response of plants modified for GRXS14 and GRXS16 expression to oxidative stress}

Studies using plate-grown plantlets or leaf disks suggested a role of GRXS14 and GRXS16 in oxidative stress responses (Cheng et al. 2006; Guo et al. 2010). Therefore, we first exposed 
273

274

275

276

277

278

279

soil-grown GRX-modified plants from sowing to high light and long photoperiod, conditions affecting redox homeostasis (Laugier et al. 2010) and impairing the development of grxS17 plants (Knuesting et al. 2015). After 3 weeks, the rosette weights of plants overexpressing GRXS14 or of grxS14 plants knockdown for GRXS16 were on average $25 \%$ higher and $22 \%$ lower, respectively, than that of WT (Fig. S5a), indicating that high light does not further affect the growth of modified lines compared to standard conditions (Fig. 3a). Again, both overexpressing lines displayed significantly lower chlorophyll contents (Fig. S5b). In another series of experiments, we exposed plants to acute photooxidative stress, very high light at $6^{\circ} \mathrm{C}$, conditions leading to photobleaching of thylakoids (Rey et al. 2007). After 11 days, no difference was observed in modified lines compared to WT, none of them showing severe damage (Fig. S5c). Finally, we incubated leaf disks on methyl viologen and measured the maximal PSII activity. Disks from plants deficient in GRXS14 displayed lower, but nonsignificantly different, $\mathrm{F}_{\mathrm{v}} / \mathrm{F}_{\mathrm{m}}$ values compared to WT and GRXS14-overexpressing plants (Fig. S5d). Similarly, when incubating disks from plants modified for GRXS16 expression on methyl viologen, no significant difference in $F_{v} / F_{m}$ values was noticed (Fig. S6a). Taken collectively, these data indicate that GRXS14 and GRXS16 do not likely play essential roles in vivo in the protection of photosynthetic structures against oxidative damage.

\section{Response of plants modified for GRXS14 and GRXS16 expression to osmotic stress}

Overexpression of tomato GRXS16 in Arabidopsis has been reported to confer tolerance to $\mathrm{NaCl}$ both in in vitro and in vivo conditions (Guo et al. 2010). We thus investigated the response of the lines generated in this work to high salinity by first watering soil-grown plants with a solution containing $0.15 \mathrm{M} \mathrm{NaCl}$ during one month. Plant growth was considerably reduced since the rosette weight at the end of the experiment was around $0.7 \mathrm{~g}$ (Fig. 3d) compared to $2.3 \mathrm{~g}$ in control conditions (Fig. 3a). Most interestingly, similar rosette weights 
were measured for WT, GRXS14-overexpressing (O1 and O2) and grxS14 plants knockdown or not for GRXS16 (K, KI1 and KI2) in contrast to standard conditions (Fig. 3a, d). While no change in the maximal PSII activity was observed (Fig. S3b), higher chlorophyll contents were measured compared to control conditions, but consistent with the data acquired in other environmental conditions, lines overexpressing GRXS14 (O1 and O2) displayed substantially lower pigment levels in well-expanded leaves than other lines. (Fig. 3c, f). Strikingly, a loss of pigments was observed in very young leaves of $\mathrm{O} 1$ and $\mathrm{O} 2$ plants, which were also almost necrotic in contrast to what was observed in other genotypes (Fig. 3e). When performing a similar salt treatment on lines modified for GRXS16 expression, plant growth and chlorophyll content were not impaired compared to WT while the PSII photosynthetic efficiency was significantly reduced in plants lacking GRXS16, but not in overexpressing ones (Fig. S6b-e).

To further investigate the effects of high salt, we carried out incubation experiments using leaf disks. On water, no difference with regard to maximal PSII activity and chlorophyll content was noticed between WT and lines modified for the expression of GRXS14 and/or GRXS16, except for the $\mathrm{O} 2$ line (Figs. 4a, S3c, S6a). In contrast, the $\mathrm{F}_{\mathrm{v}} / \mathrm{F}_{\mathrm{m}}$ values $(\mathrm{ca} 0.30)$ of discs from grxS14 plants knockdown or not for GRXS16 (K, KI1 and KI2) were significantly lower than that of WT (0.40) upon incubation on $0.15 \mathrm{M} \mathrm{NaCl}$ in the light phytotron conditions for $48 \mathrm{~h}$ and higher (0.46) in disks from GRXS14-overexpressing $\mathrm{O} 1$ and $\mathrm{O} 2$ plants (Fig. S3c). Of note, all lines exhibited a similar chlorophyll content following the incubation on salt in the light for 2 days (Fig. 4a). To determine whether the PSII phenotype trait of GRXS14-modified plants was related to osmotic stress or to $\mathrm{NaCl}$ toxicity, we incubated discs on $0.3 \mathrm{M}$ mannitol or $0.15 \mathrm{M} \mathrm{KCl}$ (Fig. S3c). Mannitol and $\mathrm{KCl}$ induced slight and strong, respectively, decreases in PSII activity, but to the same extent in GRXS14modified lines and WT. These data reveal that the higher sensitivity to $\mathrm{NaCl}$ of leaf disks from GRXS14-deficient plants is very likely associated with $\mathrm{Na}^{+}$toxicity. We then performed 
the $\mathrm{NaCl}$ treatment in the dark. The treatment on water strongly and similarly impaired the PSII activity in all lines (Fig S3d), but barely affected the chlorophyll content (Fig. 4b). In the presence of $\mathrm{NaCl}$, grxS14 plants knockdown or not for GRXS16 (K, KI1 and KI2) exhibited $\mathrm{F}_{\mathrm{v}} / \mathrm{F}_{\mathrm{m}}$ values decreased to 0.4 and chlorophyll contents reduced to $10 \mu \mathrm{g} . \mathrm{cm}^{-2}$ (compared to 0.65 and $17 \mu \mathrm{g} . \mathrm{cm}^{-2}$, respectively, in WT) (Figs. S3d, 4b). Altogether, these data reveal that GRXS14 plays important, but complex roles in the responses to $\mathrm{NaCl}$, since contrasted results were obtained when using whole plants or leaf disks.

\section{Response of plants modified for GRXS14 and GRXS16 expression to dark exposure}

The strong decrease in chlorophyll content in grxS14 leaf disks exposed to $\mathrm{NaCl}$ in the dark (Fig. 4b), coupled to the observed decrease in GRXS14 transcript level in senescent leaves (eFP Browser; http://bar.utoronto.ca/efp/cgi-bin/efpWeb.cgi), prompted us to study the response to dark exposure of GRXS14-modified lines. First, we incubated disks on water in the dark for 3 to 5 days (Fig. 4c). Following 3 days, almost no decrease was noticed in the chlorophyll content of WT and GRXS14-overexpressing plants compared to light conditions whereas a marked reduction, from $c a 25$ to $20 \mu \mathrm{g} . \mathrm{cm}^{-2}$, occurred in disks from GRXS14deficient K, KI1 and KI2 lines (Figs. 4a, c). Thereafter, a dramatic decrease in pigment content was observed in all lines after 4 or 5 days in the dark. Of note, this decrease was much more pronounced in grxS14 lines down-regulated or not for GRXS16 expression (K, KI1 and KI2), since they showed a twice lower chlorophyll content than WT and GRXS14overexpressing plants (Figs. S7a, 4c). We then covered leaves with aluminum foil for 8 days on entire plants in standard light conditions. Such a treatment has been reported to induce senescence (Weaver and Amasino, 2001). Most dark-exposed WT leaves remained pale green and some exhibited limited yellowing (Fig. 4d). In these leaves, a lower chlorophyll content was measured compared to control conditions (17.0 vs $26.5 \mu \mathrm{g} . \mathrm{cm}^{-2}$, Figs. 3c, 4e). A very 
348 similar pigment level was measured in covered leaves from GRXS14-overexpressing plants.

349 In contrast, a high proportion (50\%) of dark-treated leaves from grxS14 plants down-regulated 350 or not for GRXS16 (K, KI1 and KI2) displayed pronounced yellowing (Fig. 4d).

351 Consequently, their pigment content ( 8 to $10 \mu \mathrm{g} . \mathrm{cm}^{-2}$ ) was much lower than that in leaves 352 from WT and from KI1 plants restored for GRXS14 expression (KI1-R1 and -R2) subjected to 353 the same treatment ( $\mathrm{ca} 17 \mu \mathrm{g} . \mathrm{cm}^{-2}$ ) (Figs. 4e, S2c). Experiments on dark-exposed disks and 354 leaves of lines modified for GRXS16 expression did not reveal any difference in the pigment 355 content compared to WT (Fig. S7b-e), clearly demonstrating that plants deficient in GRXS14, 356 but not GRXS16, are sensitive to prolonged darkness.

357

Redox status of GRXS14 in leaf extracts

359

The redox status of GRXS14 in planta, here in leaves, is an important question to ask as this is likely directly connected to its reductase activity. This was first analyzed by preparing extracts in the absence of reductant and performing non-reducing SDS-PAGE followed by Western blot analysis. In this experiment, a much lower protein amount from the GRXS14overexpressing line $(5 \mu \mathrm{g})$ was loaded compared to that from grxS14 (K) and WT lines (30 $\mu \mathrm{g})$ to avoid signal saturation. In this case, the serum against GRXS14 revealed several specific bands in protein extracts from WT and GRXS14-overexpressing plants (Fig. 5a). Indeed, two bands very likely corresponding to monomers displaying different redox status were observed at $c a 12 \mathrm{kDa}$. A band at $c a 24 \mathrm{kDa}$ that could correspond to a covalent GRXS14 dimer or a GRXS14-partner complex was clearly detected in overexpressing plants. Another band at $c a 70 \mathrm{kDa}$, which might correspond to an oligomer containing GRXS14 alone or linked to partner(s), was specifically revealed in extracts from overexpressing plants (Fig. 5a). The latter band was also faintly revealed in WT protein extracts. 
The GRXS14 redox status was then analyzed by carrying out alkylation experiments

373

374

375

376

377 using mPEG-maleimide-2000 that forms stable thioether bonds with thiol groups and allows the detection of reduced cysteines due to a $2-\mathrm{kDa}$ size increase per free alkylated thiol. Following extraction in non-reducing conditions, incubation with this compound and Western analysis, the 12-kDa forms were almost no more visible in WT and GRXS14-overexpression leaf samples (Fig. 5b). Instead, two bands at ca 14 and $18 \mathrm{kDa}$ were clearly and specifically observed, these bands being absent in samples from grxS14 (K) plants (Fig. 5b). Note that in this experiment, the loading per lane of extracts from overexpressing plants was also much lower $(15 \mu \mathrm{g})$ than that of WT plants $(100 \mu \mathrm{g})$ to avoid saturated signal levels for the specific 14- and 18-kDa bands. Considering that GRXS14 contains 3 cysteines, the 2 and $6 \mathrm{kDa}$ mass increases likely correspond to the alkylation of one and three thiols, respectively, and allow distinguishing a monomeric form likely displaying one intramolecular disulfide and one reduced cysteine, from a fully reduced form. Based on the signal level of the $18-\mathrm{kDa}$ band, we conclude that the completely reduced GRX form ( 3 free cysteines) is more abundant in WT extracts in control conditions. Note that, in extracts from overexpressing plants, this form was proportionally less abundant, possibly due to impaired reduction of the huge GRX amount.

This prompted us to investigate whether environmental constraints could affect the GRXS14 redox state in WT plants. In control conditions, the proportion of the 3-thiol form was in the range of $60 \%$ with the remaining corresponding to the oxidized $14-\mathrm{kDa}$ form. Interestingly, the ratio was significantly lower in plants exposed to $\mathrm{NaCl}$ for 1 month (ca $40 \%$ ) and in leaves covered with aluminum for 8 days ( $c a 50 \%)$ and no variation in this proportion was noticed in plants grown in high light (Fig. 5c-f). These data indicate that the in planta GRXS14 redox status is modified during specific environmental constraints such as high salinity or prolonged darkness. 


\section{Abundance of plastidial redoxins and of proteins involved in $\mathrm{Fe}-\mathrm{S}$ cluster biogenesis in} response to dark exposure

We previously showed that leaves from GRXS14-deficient lines displayed accelerated yellowing upon prolonged darkness (Fig. 4) and that this treatment led to GRX oxidation in WT (Fig. 5). We then analyzed the GRXS14 abundance in dark-exposed WT leaves and observed a reduced protein amount (- 25\%) compared to control, no change being observed regarding GRXS16 abundance (Fig. 6a,b). In parallel, we investigated the amount of some plastidial thiol reductases involved in redox homeostasis maintenance, notably PRXs which detoxify $\mathrm{H}_{2} \mathrm{O}_{2}$ and organic peroxides and methionine sulfoxide reductases (MSRs) which repair oxidized proteins (Vieira dos Santos and Rey, 2006). Regarding PRXs, no change was observed for PRXIIE and PRXQ (Fig. 6a), the abundance of which can vary in response to abiotic constraints (Gama et al. 2007). An increased amount (+20\%) of 2-Cys PRX, the most abundant PRX, was noticed in dark-treated leaves and most interestingly, its overoxidized form was much more abundant $(+100 \%)$ (Fig. 6a,b). The plastidial MSRA4 displays two redox forms, the proportion of which varies depending on light intensity (Vieira dos Santos et al. 2005). Upon darkness, no substantial variation was observed in both reductase amount and redox status. The analysis was then extended to proteins participating in the biogenesis of FeS proteins in plastids, a process in which GRXS14 could be involved (Couturier et al. 2013). Our data showed that the dark treatment led to strong decreases in the abundance of NFU2 (55\%), NFU3 (- 60\%), SUFA1 (- 33\%) and SUFB (- 72\%), but not of NFS2, SUFE1 and BOLA4 (Fig. 6a,b). Taken collectively, these data revealed that prolonged darkness results on one hand in substantial changes in the plastidial redox homeostasis, as illustrated by the high amount of overoxidized 2-Cys PRX form and the modified redox status of GRXS14, and on the other hand in strong decreases in the abundance of several key components of the chloroplastic Fe-S cluster assembly machinery. 


\section{Discussion}

\section{A single plastidial CGFS-GRX is sufficient for proper growth of $\boldsymbol{A}$. thaliana plants}

This study aimed at clarifying and comparing the physiological functions of the two plastidial class-II GRXs, GRXS14 and GRXS16, in A. thaliana. In standard conditions, plants lacking a single GRX did not show any obvious phenotype whereas the rosette weight of lines knockout for GRXS14 and knockdown for GRXS16 was reduced by more than $20 \%$, indicating that a protein expression patterns (Fig. 1) and the presence of a regular GRX domain in both. On the other hand, plants overexpressing GRXS14, but not GRXS16, displayed enhanced growth in all environmental conditions tested, except upon high salinity (Figs. 3, S5). Accordingly, overexpression of a fern GRXS14 orthologue in Arabidopsis led to either comparable or in fact numerous examples of thiol reductases being required for plant growth or development particularly upon environmental conditions that alter redox homeostasis (Rouhier et al. 2015).

This is the case of plastidial TRXs y or methionine sulfoxide reductases $\mathrm{B}$, the deficiency of which results in reduced growth under high light conditions (Laugier et al. 2010; Laugier et al. 2013). Regarding the GRX superfamily, several members, including the two other class-II

442 GRXs (GRXS15 and GRXS17), are important for plant development (Xing and Zachgo, 2005; Riondet et al. 2012; Knuesting et al. 2015; Moseler et al. 2015; Yang et al. 2015) or involved in responses to environmental constraints (Laporte et al. 2012). A strongly reduced 
446 observed in overexpression lines (Laporte et al. 2012), in contrast to what was observed here

447 for GRXS14. To conclude, these data indicate that plastidial class-II GRXs fulfil important 448 roles in the control of growth in Arabidopsis, the overexpression of GRXS14 being even 449 beneficial in various environmental conditions.

\section{GRXS14 participates in chlorophyll maintenance}

GRXS14 and GRXS16 have been proposed to participate in responses to oxidative stress based on data mostly gained either using leaf discs or in vitro plantlets (Cheng et al. 2006; Guo et al. 2010) or upon ectopic expression in Arabidopsis (Sundaram et al. 2009; Guo et al. 2010). Having noticed the substantial variation in GRXS14 abundance upon light or osmotic constraints (Fig. 1b), our goal was to explore the phenotype of GRXS14-modified lines in these conditions. In comparison with previously published reports, constraints have been mainly applied on soil-grown plants in relatively long-term experiments. When applying conditions generating severe oxidative stress, no susceptibility of lines deficient in GRXS14 and/or GRXS16 was observed (Figs. S5, S6). This might appear contradictory to the root growth reduction observed in vitro upon $\mathrm{H}_{2} \mathrm{O}_{2}$ treatment (Cheng et al. 2006), but it is likely that the heterotrophic metabolism in vitro results in $\mathrm{H}_{2} \mathrm{O}_{2}$ responses non representative of what happens in more physiological conditions.

When investigating the responses to salt of lines modified for GRXS16, no difference was noticed in short-term experiments performed on disks. But GRXS16-lacking plants watered with $150 \mathrm{mM} \mathrm{NaCl}$ showed reduced PSII activity although this had no impact on the rosette weight (Fig. S6). This is in agreement with the better tolerance of Arabidopsis plants overexpressing tomato GRXS16 exposed to $300 \mathrm{mM} \mathrm{NaCl}$ (Guo et al. 2010). Regarding lines modified for GRXS14 expression, the response to salt greatly varied as a function of the type of material used. Using leaf discs, plants lacking GRXS14 exhibited a lower tolerance to 
$471 \mathrm{NaCl}$ as illustrated by a decrease in PSII photosynthetic activity (Fig. S3c). Consistently, discs 472 from plants overexpressing GRXS14 better tolerated the $\mathrm{NaCl}$ treatment (Fig. S3c). On the 473 other hand, when analyzing salt-treated plants, GRXS14 overexpression was detrimental as 474 inferred from the measurement of substantially reduced chlorophyll content and the presence 475 of yellowish young developing leaves (Fig. 3e, f). These data clearly highlight the complex/subtle roles that GRXS14 could play in response to salt and the importance of the type of material used for investigating physiological responses of plants. The observed discrepancy might be explained by the fact that leaf discs and whole plants do undoubtedly respond in a distinct manner to the salt application, i.e. stomatal entry in the case of discs and root absorption in soil-grown plants. Hence, the first cells perceiving the high sodium concentration are distinct, probably leading to contrasted responses.

In experiments promoting dark-induced senescence, the responses of disks and covered leaves showed consistency. Indeed, in both cases, a strong decrease in chlorophyll content was observed in plants deficient in GRXS14 compared to WT and overexpressing lines (Fig. 4), while there was no effect in lines modified for GRXS16 expression (Fig. S7). Of possible importance is that in disks this phenotype trait was exacerbated in the presence of salt (Fig. 4b). This additive effect could point to possible distinct and cumulating GRXS14 functions related to light and sodium stresses. Coupled with the observation that GRXS14overexpressing lines display a significantly lower chlorophyll content in control, high salt or high light conditions (Figs. 3, S5), we conclude that GRXS14 plays critical and specific roles in the maintenance of chlorophyll content.

\section{What is the molecular role of GRXS14 in chlorophyll maintenance?}

In the last years, the involvement of several types of thiol reductases in the regulation of chlorophyll content has been reported. For instance, plants lacking plastidial methionine 
sulfoxide reductases B exhibit a reduced pigment level in high light conditions (Laugier et al. 2010). MSRBs have been proposed to prevent oxidative damage in the system targeting proteins in chlorophyll antennae. In other respects, the stability and/or activity of enzymes involved in tetrapyrrole biogenesis is finely controlled at the post-translational level by thiolbased redox mechanisms (Richter and Grimm, 2013). Indeed, the NADPH-dependent TRX reductase $\mathrm{C}$ (NTRC), which has a TRX domain, regulates the redox status of magnesium protoporphyrin IX methyltransferase (Richter et al. 2013). Accordingly, plants deficient in NTRC show pale leaves and impaired growth depending on light and photoperiod conditions (Toivola et al. 2013). So far, a role of GRXs in the redox regulation of tetrapyrrole biosynthesis has not been demonstrated, but proteomic studies in Chlamydomonas reinhardtii and Synechocystis PCC6803 suggested that several enzymes of this pathway could be prone to glutathionylation, an oxidative post-translational modification quite specifically reversed by GRXs (Michelet et al. 2008; Zaffagnini et al. 2012; Chardonnet et al. 2015). Of note, the senescence process is accompanied by increased ROS formation that could promote glutathionylation (Zimmermann and Zentgraf, 2005). Here, the impaired redox homeostasis in dark-treated leaves is revealed by the increased level of overoxidized 2-Cys PRX (Fig. 6). Most importantly, the GRXS14 redox status varied toward a more oxidized intramolecular disulfide-containing form (Fig. 5) upon dark exposure and upon salt treatment, which also impairs redox homeostasis (Munns and Tester, 2008), arguing for a redox regulatory function. Indeed, the formation of an intramolecular disulfide could constitute a regular intermediate recycling state for class-II GRXs upon deglutathionylation or disulfide bond reduction in target proteins (Zaffagnini et al. 2008).

Alternatively, the GRXS14 function could be related to its participation in the maturation of $\mathrm{Fe}-\mathrm{S}$ proteins based on its capacity to bind a labile $\left[\mathrm{Fe}_{2} \mathrm{~S}_{2}\right]$ cluster and to transfer it to acceptor proteins (Bandyopadhyay et al. 2008). Evidence for such a 
521 physiological role has been only reported for the mitochondrial GRXS15 isoform (Moseler et 522 al. 2015; Ströher et al. 2016). Several key enzymes in chlorophyll metabolism incorporate an 523 Fe-S center (Balk and Pilon, 2011): chlorophyllide $a$ oxygenase (CAO) and 7-hydroxymethyl 524 chlorophyll $a$ reductase (HCAR), which are involved in the conversion between chlorophylls $525 a$ and $b$ (Oster et al. 2000), and pheophorbide $a$ oxygenase (PAO), which is involved in 526 chlorophyll breakdown (Pruzinska et al. 2003). Moreover, other proteins in this biosynthetic 527 pathway require the $\left[\mathrm{Fe}_{2} \mathrm{~S}_{2}\right]$-containing $\mathrm{FDX}$ for functioning (Richter \& Grimm, 2013). 528 Accordingly, Arabidopsis mutants deficient in subunits of the central $\mathrm{SUFBC}_{2} \mathrm{D}$ scaffold 529 complex of the plastidial Fe-S cluster assembly machinery show a pale phenotype, 530 accompanied by lower PAO, HCAR and FDX amounts (Hu et al. 2017). Here, we obtained 531 evidence that the protein levels of most SUF components, SUFB, NFU2, NFU3, SUFA1 and 532 GRXS14, but not those of the very early acting NFS2 and SUFE1 proteins, are substantially 533 reduced in WT leaves upon dark-induced senescence (Fig. 6). This could indicate a general 534 down-regulation of the SUF machinery, in agreement with the rapid shut down of chloroplast 535 metabolism in this condition (Chrobok et al. 2016). The findings that GRXS14 deficiency 536 results in drastically reduced chlorophyll content in dark conditions and that its 537 overexpression is associated with both modified chlorophyll content and reduced NFU2 and SUFE1 amounts, strongly suggest that GRXS14 acts within the SUF machinery to regulate chlorophyll metabolism in relation with environmental conditions, and that its deficiency or a 540 non-physiological amount impair the functioning of this machinery. Which step(s) is(are) 541 controlled by GRXS14 in the chlorophyll biosynthetic or degradation pathways and whether 542 its redox status modifies the Fe-S cluster binding properties remain to be studied. 


\section{Acknowledgements}

547 We are very grateful to the Groupe de Recherche Appliquée en Phytotechnologie, Patricia

548 Henri and Dr Delphine Cerveau (CEA, DRF, BIAM) for assistance with growth chambers and 549 preparation of plant extracts. We would like also to thank Dr Michel Havaux and Dr 550 Dominique Rumeau (UMR 7265, CEA-CNRS-AMU) for advice regarding photosynthetic 551 measurements and transformation procedures and Dr Olivier Keech (Umea University), Dr. 552 Jérémy Couturier and Prof Jean-Pierre Jacquot (University of Lorraine) for critical reading of 553 the manuscript. This work was supported by the Agence Nationale de la Recherche (ANR), 554 grant no. 2010 BLAN-1616. Moreover, the UMR 1136 is supported by a grant overseen by 555 ANR as part of the "Investissements d'Avenir" program (ANR-11-LABX-0002-01, Lab of 556 Excellence ARBRE).

557

558

559

560

561

562

563

564

565

566

567

568

569

570 


\section{References}

Balk J \& Pilon M. (2011) Ancient and essential: the assembly of iron-sulfur clusters in plants. Trends in Plant Science 16, 218-226.

\section{Bandyopadhyay S., Gama F., Molina-Navarro M.M., Gualberto J.M., Claxton R., Naik} SG., ..., Rouhier N. (2008) Chloroplast monothiol glutaredoxins as scaffold proteins for the assembly and delivery of [2Fe-2S] clusters. EMBO Journal 27, 1122-1133.

Cerveau D., Ouahrani D., Marok MA., Blanchard L. \& Rey P. (2016) Physiological relevance of plant 2-Cys peroxiredoxin overoxidation level and oligomerization status., Plant; Cell and Environment 39, 103-119.

Chardonnet S., Sakr S., Cassier-Chauvat C., Le Maréchal P., Chauvat F., Lemaire SD. \& Decottignies P. (2012) First proteomic study of S-glutathionylation in cyanobacteria. Journal of Proteome Research 14, 59-71.

Chaubal R., Anderson J.R., Trimnell M.R., Fox T.W., Albertsen M.C. \& Bedinger P. (2003) The transformation of anthers in the mscal mutant of maize. Planta 216, 778-788.

Cheng N.H., Liu J.Z., Brock A., Nelson R.S. \& Hirschi K.D. (2006) AtGRXcp, an Arabidopsis chloroplastic glutaredoxin, is critical for protection against protein oxidative damage. Journal of Biological Chemistry 281, 26280-26288.

Cheng N.H., Liu J.Z., Liu X., Wu Q., Thompson SM., Lin J., ..., Hirschi K.D. (2011) Arabidopsis monothiol glutaredoxin., AtGRXS17, is critical for temperature-dependent postembryonic growth and development via modulating auxin response. Journal of Biological Chemistry 286, 20398-20406.

Chrobok D., Law S.R., Brouwer B., Lindén P., Ziolkowska A., Liebsch D., Narsai R., ..., Keech O. (2016) Dissecting the metabolic role of mitochondria during developmental leaf senescence. Plant Physiology 172, 2132-2153. 
Clough S.J. \& Bent A.F. (1998) Floral dip: a simplified method for Agrobacterium-mediated transformation of Arabidopsis thaliana. Plant Journal 16, 735-43.

Couturier J., Jacquot J.P. \& Rouhier N. (2009) Evolution and diversity of glutaredoxins in photosynthetic organisms. Cellular and Molecular Life Sciences 66, 2539-2557.

Couturier J., Touraine B., Briat J.F., Gaymard F. \& Rouhier N. (2013) The iron-sulfur cluster assembly machineries in plants: current knowledge and open questions. Frontiers in Plant Science 4, 259.

Gama F., Brehelin C., Gelhaye E., Meyer Y., Jacquot J.P., Rey P. \& Rouhier N. (2008) Functional analysis and expression characteristics of chloroplastic Prx IIE. Physiologia Plantarum 133, 599-610.

Guo Y., Huang C., Xie Y., Song F. \& Zhou X. (2010) A tomato glutaredoxin gene S1GRX1 regulates plant responses to oxidative, drought and salt stresses. Planta 232, 1499-1509.

Havaux M., Bonfils J.P., Lutz C. \& Niyogi K.K. (2000) Photodamage of the photosynthetic apparatus and its dependence on the leaf developmental stage in the npql Arabidopsis mutant deficient in the xanthophyll cycle enzyme violaxanthin de-epoxidase. Plant Physiology 124, 273-284.

Hong L., Tang D., Zhu K., Wang K., Li M. \& Cheng Z. (2012) Somatic and reproductive cell development in rice anther is regulated by a putative glutaredoxin. Plant Cell 24, 577588.

Hu X., Kato Y., Sumida A., Tanaka A. \& Tanaka R. (2017) The SUFBC2D complex is required for the biogenesis of all major classes of plastid Fe-S proteins. Plant Journal. 90, $235-248$.

Iñigo S., Durand A.N., Ritter A., Le Gall S., Termathe M., Klassen R., ..., Goossens A. (2016) Glutaredoxin GRXS17 associates with the cytosolic iron-sulfur cluster assembly pathway. Plant Physiology 172, 858-873. 
620

621

622

623

624

625

626

627

628

629

630

631

632

633

634

635

636

637

638

639

640

641

642

643

644

Kelliher T. \& Walbot V. (2012) Hypoxia triggers meiotic fate acquisition in maize. Science 337, 345-348.

Knuesting J., Riondet C., Maria C., Kruse I., Bécuwe N., König N., ..., Rey P. (2015) Arabidopsis glutaredoxin S17 and its partner NF-YC11/NC2 $\alpha$ contribute to maintenance of the shoot apical meristem under long-day photoperiod. Plant Physiology 167, 1643-1658.

La Camera S., L'Haridon F., Astier J., Zander M., Abou-Mansour E., Page G., ..., Lamotte O. (2011) The glutaredoxin ATGRXS13 is required to facilitate Botrytis cinerea infection of Arabidopsis thaliana plants. Plant Journal 68, 507-519.

Laporte D., Olate E., Salinas P., Salazar M., Jordana X. \& Holuigue L. (2012) Glutaredoxin GRXS13 plays a key role in protection against photooxidative stress in Arabidopsis. Journal of Experimental Botany 63, 503-515.

Laugier E., Tarrago L., Courteille A., Innocenti G., Eymery F., Rumeau D., IssakidisBourguet E. \& Rey P. (2013) Involvement of thioredoxin y2 in the preservation of leaf methionine sulfoxide reductase capacity and growth under high light. Plant, Cell and Environment 36, 670-682.

Laugier E., Tarrago L., Vieira Dos Santos C., Eymery F., Havaux M. \& Rey P. (2010) Arabidopsis thaliana plastidial methionine sulfoxide reductases B, MSRBs, account for most leaf peptide MSR activity and are essential for growth under environmental constraints through a role in the preservation of photosystem antennae. Plant Journal 61, 271-282.

Lichtenthaler H.K. (1987) Chlorophylls and carotenoids: pigments of photosynthetic biomembranes. Methods in Enzymology 148, 350-382.

Lillig C.H., Berndt C., Vergnolle O., Lonn M.E., Hudemann C., Bill E. \& Holmgren A. (2005) Characterization of human glutaredoxin 2 as iron-sulfur protein: a possible role as redox sensor. Proceedings of the National Academy of Sciences USA 102, 8168-8173. 
645 Liu X., Liu S., Feng Y., Liu J.Z., Chen Y., Pham K., ..., Cheng N. (2013) Structural 646 insights into the N-terminal GIY-YIG endonuclease activity of Arabidopsis glutaredoxin 647 AtGRXS16 in chloroplasts. Proceedings of the National Academy of Sciences USA 110, $648 \quad 9565-9570$.

649

650

651

652

653

654

655

656

657

658

659

660

661

662

663

664

665

666

667

668

Meyer Y., Buchanan B.B., Vignols F. \& Reichheld J.P. (2009) Thioredoxins and glutaredoxins: unifying elements in redox biology. Annual Review of Genetics 43, 335367.

Michelet L., Zaffagnini M., Vanacker H., Le Maréchal P., Marchand C., Schroda M., Lemaire S.D. \& Decottignies P. (2008) In vivo targets of S-thiolation in Chlamydomonas reinhardtii. Journal of Biological Chemistry 283, 21571-21578.

Moseler A., Aller I., Wagner S., Nietzel T., Przybyla-Toscano J., Mühlenhoff U., ..., MeyerA.J. (2015) The mitochondrial monothiol glutaredoxin S15 is essential for ironsulfur protein maturation in Arabidopsis thaliana. Proceedings of the National Academy of Sciences USA 112, 13735-13740.

Munns R. \& Tester M. (2008) Mechanisms of salinity tolerance. Annual Review of Plant Biology 59, 651-681.

Ndamukong I., Abdallat A.A., Thurow C., Fode B., Zander M., Weigel R. \& Gatz C. (2007) SA-inducible Arabidopsis glutaredoxin interacts with TGA factors and suppresses JA-responsive PDF1.2 transcription. Plant Journal 50, 128-139.

Oster U., Tanaka R., Tanaka A. \& Rüdiger W. (2000) Cloning and functional expression of the gene encoding the key enzyme for chlorophyll b biosynthesis (CAO) from Arabidopsis thaliana. Plant Journal 21, 305-310.

Pruzinská A., Tanner G., Anders I., Roca M. \& Hörtensteiner S. (2003) Chlorophyll breakdown: pheophorbide a oxygenase is a Rieske-type iron-sulfur protein encoded by the 
669

670

671

672

673

674

675

676

677

678

679

680

681

682

683

684

685

686

687

688

689

690

691

692

693

accelerated cell death 1 gene. Proceedings of the National Academy of Sciences USA 100, 15259-15264.

Rey P., Bécuwe N., Barrault M.B., Rumeau D., Havaux M., Biteau B. \& Toledano M.B. (2007) The Arabidopsis thaliana sulfiredoxin is a plastidic cysteine-sulfinic acid reductase involved in photooxidative stress response. Plant Journal 49, 505-514.

Rey P., Cuine S., Eymery F., Garin J., Court M., Jacquot J.P., Rouhier N. \& Broin M. (2005) Analysis of the proteins targeted by CDSP32, a plastidic thioredoxin participating in oxidative stress responses Plant Journal 41, 31-42.

Richter A.S. \& Grimm B. (2013) Thiol-based redox control of enzymes involved in the tetrapyrrole biosynthesis pathway in plants. Frontiers in Plant Science. 4, 371.

Richter A.S., Peter E., Rothbart M., Schlicke H., Toivola J., Rintamäki E. \& Grimm B. (2013) Posttranslational influence of NADPH-dependent thioredoxin reductase C on enzymes in tetrapyrrole synthesis. Plant Physiology 162, 63-73.

Riondet C., Desouris J.P., Montoyan J.G., Chartier Y., Meyer Y. \& Reichheld J.P. (2012) A dicotyledon-specific glutaredoxin GRXC1 family with dimer-dependent redox regulation is functionally redundant with GRXC2. Plant, Cell and Environment 35, 360373.

Rodriguez-Manzaneque M.T., Tamarit J., Belli G., Ros J. \& Herrero E. (2002) Grx5 is a mitochondrial glutaredoxin required for the activity of iron/sulfur enzymes. Molecular Biology of the Cell 13, 1109-1121.

Rouhier N., Cerveau D., Couturier J., Reichheld J.P. \& Rey P. (2015) Involvement of thiol-based mechanisms in plant development. Biochimica et Biophysica Acta 1850, 14791496.

Rouhier N., Couturier J. \& Jacquot J.P. (2006) Genome-wide analysis of plant glutaredoxin systems. Journal of Experimental Botany 57, 1685-1696. 
694 Rouhier N., Couturier J., Johnson M.K. \& Jacquot J.P. (2010) Glutaredoxins: roles in 695 iron homeostasis. Trends in Bochemical Sciences 35, 43-52.

696 Rouhier N., Lemaire S.D. \& Jacquot J.P. (2008) The role of glutathione in photosynthetic 697 organisms: emerging functions for glutaredoxins and glutathionylation. Annual Review of $698 \quad$ Plant Biology 59, 143-166.

699

700

701

702

703

704

705

706

707

708

709

710

711

712

713

714

715

716

717

Rouhier N., Unno H., Bandyopadhyay S., Masip L., Kim S.K., Hirasawa M., ..., Jacquot J.P. (2007) Functional, structural, and spectroscopic characterization of a glutathioneligated [2Fe-2S] cluster in poplar glutaredoxin C1 Proceedings of the National Academy of Sciences USA 104, 7379-7384.

Ströher E., Grassl J., Carrie C., Fenske R., Whelan J. \& Millar A.H. (2016) Glutaredoxin S15 is involved in Fe-S cluster transfer in mitochondria influencing lipoic acid-dependent enzymes, plant growth and arsenic tolerance in Arabidopsis. Plant Physiology 170, 12841299.

Sundaram S. \& Rathinasabapathi B. (2010) Transgenic expression of fern Pteris vittata glutaredoxin PvGrx5 in Arabidopsis thaliana increases plant tolerance to high temperature stress and reduces oxidative damage to proteins. Planta 231, 361-369.

Sundaram S., Wu S., Ma L.Q. \& Rathinasabapathi B. (2009) Expression of a Pteris vittata glutaredoxin PvGRX5 in transgenic Arabidopsis thaliana increases plant arsenic tolerance and decreases arsenic accumulation in the leaves. Plant, Cell and Environment 32, 851858.

Toivola J., Nikkanen L., Dahlström K.M., Salminen T.A., Lepistö A., Vignols F. \& Rintamäki E. (2013) Overexpression of chloroplast NADPH-dependent thioredoxin reductase in Arabidopsis enhances leaf growth and elucidates in vivo function of reductase and thioredoxin domains. Frontiers in Plant Science 4, 1-18. 
Vieira Dos Santos C., Cuiné S., Rouhier N. \& Rey P. (2005) The Arabidopsis plastidic methionine sulfoxide reductases B proteins: sequence and activity characteristics, comparison of the expression with plastidic methionine sulfoxide reductase A and induction by photooxidative stress. Plant Physiology 138, 909-922.

Vieira Dos Santos C. \& Rey P. (2006) Plant thioredoxins are key actors in oxidative stress response. Trends in Plant Sciences 11, 329-334.

Weaver L.M. \& Amasino R.M. (2001) Senescence is induced in individually darkened Arabidopsis leaves, but inhibited in whole darkened plants. Plant Physiology 127, 876886.

Xing S., Rosso M.G. \& Zachgo S. (2005) ROXY1, a member of the plant glutaredoxin family, is required for petal development in Arabidopsis thaliana. Development 132, 1555 1565.

Xing S. \& Zachgo S. (2008) ROXY1 and ROXY2, two Arabidopsis glutaredoxin genes, are required for anther development. Plant Journal 53, 790-801.

Yang F., Bui H.T., Pautler M., Llaca V., Johnston R., Lee B.H., ..., Jackson D. (2015) A maize glutaredoxin gene, Abphyl2, regulates shoot meristem size and phyllotaxy. Plant Cell 27, 121-131.

Zaffagnini M., Bedhomme M., Groni H., Marchand C.H., Puppo C., Gontero B., ..., Lemaire S.D. (2012) Glutathionylation in the photosynthetic model organism Chlamydomonas reinhardtii: a proteomic survey. Molecular Cell Proteomics 11, M111.014142.

Zaffagnini M., Michelet L., Massot V., Trost P. \& Lemaire S.D. (2008) Biochemical characterization of glutaredoxins from Chlamydomonas reinhardtii reveals the unique properties of a chloroplastic CGFS-type glutaredoxin. Journal of Biological Chemistry 283, $8868-8876$. 
743 Zimmermann P. \& Zentgraf U. (2005) The correlation between oxidative stress and leaf $744 \quad$ senescence during plant development. Cell and Molecular Biology Letters 10, 515-534. 
747

748

749

751

753

754
755

756

757
758

759

760

761

762

763

765

766

767

768

769

770

771

772

773

774

775

776

777

778

779

780

781

782

Table 1 Characteristics and names of Arabidopsis thaliana lines modified for the expression of GRXS14 and/or GRXS16

\begin{tabular}{|c|c|}
\hline Name & Characteristics \\
\hline WT & Col-0 \\
\hline K & grxS14, knockout for GRXS14 expression (SALK_125902), \\
\hline $\begin{array}{l}\mathrm{O} 1(\mathrm{OE}-\mathrm{S} 14) \\
\mathrm{O} 2(\mathrm{OE}-\mathrm{S} 14)\end{array}$ & $\begin{array}{l}\text { GRXS14 verexpression (CaMV-35S promoter) in WT background } \\
\text { GRXS14 } 0 \text { verexpression (CaMV-35S promoter) in WT background }\end{array}$ \\
\hline $\begin{array}{l}\text { KI1 } \\
\text { KI2 }\end{array}$ & $\begin{array}{l}\text { grxS14 } \underline{\mathbf{K}} \text { background, knockdown for } G R X S 16 \text { expression, RNA-interference } \\
\text { grxS14 } \underline{\mathbf{K}} \text { background, knockdown for } G R X S 16 \text { expression, RNA-interference }\end{array}$ \\
\hline $\begin{array}{l}\text { C2 (C-S16) } \\
\text { C4 (C-S16) }\end{array}$ & $\begin{array}{l}\text { GRXS16 co-suppression (CaMV-35S promoter) in WT background } \\
\text { GRXS16 } \text { co-suppression (CaMV-35S promoter) in WT background }\end{array}$ \\
\hline $\begin{array}{l}\mathrm{O} 4(\mathrm{OE}-\mathrm{S} 16) \\
\mathrm{O} 5(\mathrm{OE}-\mathrm{S} 14)\end{array}$ & $\begin{array}{l}G R X S 16 \text { overexpression (CaMV-35S promoter) in WT background } \\
\text { GRXS16 verexpression (CaMV-35S promoter) in WT background }\end{array}$ \\
\hline $\begin{array}{l}\text { KI1-R1 } \\
\text { KI1-R2 }\end{array}$ & 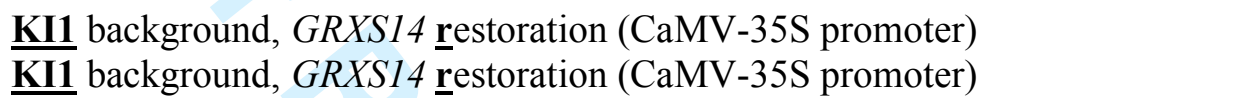 \\
\hline
\end{tabular}

.

.

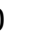


783

784

785

786

787

788

789

790

791

792

793

794

795

796

797

798

799

800

801

802

803

804

805

806

807

\section{Legends to figures}

Figure 1. Abundance of GRXS14 and GRXS16 proteins in Arabidopsis thaliana organs and in leaves upon environmental constraints.

(a) Western analysis of RubisCO, GRXS14 and GRXS16 abundance in the organs of 6-weekold Arabidopsis plants grown in short-day conditions (YL, young leaf less than $1 \mathrm{~cm}$ long; AL, adult well-expanded leaf; OL, old non-senescent leaf) and of 6-week-old plants grown in long-day conditions (R, root; St, stem; CL, cauline leaf; FB, flower bud; F, flower; GP, green pod). Seeds (Se) were harvested from plants grown for 11 weeks in long-day conditions. Gels for Western analysis were loaded as in the Coomassie blue-stained gel. (b) Western analysis of RubisCO, 2-Cys PRX, GRXS14 and GRXS16 abundance in adult leaves of Arabidopsis plants grown for 6 weeks in control conditions (C) or exposed to various light, temperature and osmotic constraints: $450 \mu \mathrm{mol}$ photons $\mathrm{m}^{-2} \cdot \mathrm{s}^{-1}$ under an 16-h photoperiod (HL-LD, high light, long day), $825 \mu \mathrm{mol}$ photons $\mathrm{m}^{-2} \cdot \mathrm{s}^{-1}$ and $30^{\circ} \mathrm{C}$ under an 8 -h photoperiod (HL-HT, high

light, high temperature), $1400 \mu \mathrm{mol}$ photons $\mathrm{m}^{-2} \cdot \mathrm{s}^{-1}, 6^{\circ} \mathrm{C}, 8-\mathrm{h}$ photoperiod for 1 week (PT, photooxidative conditions), $10^{\circ} \mathrm{C}$ for 4 weeks (LT, low temperature), watering with $150 \mathrm{mM}$ $\mathrm{NaCl}$ for 2 weeks (S, high salinity) and water deficit for 1 week (WD). Light intensity for C, LT, S and WD: $200 \mu \mathrm{mol}$ photons $\mathrm{m}^{-2} \cdot \mathrm{s}^{-1}$. Similar results were obtained when using extracts from two independent experiments.

Figure 2. Characterization of Arabidopsis lines modified in the expression of GRXS14 and/or GRXS16.

(a) and (b), PCR analysis of genomic DNA. (c) and (d), Western analysis of GRXS14 and GRXS16 abundance in leaf extracts. (a) and (c), lines modified for GRXS14 expression. (b) and (d), lines modified for GRXS14 and GRXS16 expression. WT, wild-type; K, SALK_125902 T-DNA-line knockout for GRXS14 expression; OE-S14 (O1 and O2), two 
808

809

810

811

812

813

814

815

816

817

818

819

820

821

822

823

824

825

826

827

828

829

830

831

832

independent lines overexpressing GRXS14 in the WT background; K RNAi-S16 (KI1 and KI2), two independent RNAi lines for GRXS16 expression generated in the SALK_125902 background.

Figure 3. Growth and chlorophyll content of plants modified for GRXS14 and/or GRXS16 expression in control and high salt conditions.

(a, b, c) Plants grown for 6 weeks in control conditions: moderate light (200 $\mu$ mol photons.m $\left.{ }^{2} . \mathrm{s}^{-1}\right)$ and short photoperiod $(8 \mathrm{~h})$ at $22^{\circ} \mathrm{C}$. (a) Rosette weight. (b) Photograph of KI1, WT and O1 plants. (c) Leaf chlorophyll content. (c, d, e) Plants aged of 21 days grown in control conditions and then watered with $0.15 \mathrm{M} \mathrm{NaCl}$ for 31 days in the phytotron conditions (8-h photoperiod, $200 \mu$ moles photons. $\mathrm{m}^{-2} \cdot \mathrm{s}^{-1}$ at $22^{\circ} \mathrm{C}$ ). (d) Rosette weight. (e) Photograph of WT, $\mathrm{K}, \mathrm{KI} 1, \mathrm{KI} 2, \mathrm{O} 1$ and $\mathrm{O} 2$ plants. Red arrows indicate young necrotic leaves. (f) Leaf chlorophyll content. KI1 and KI2: lines KO for GRXS14 and RNAi for GRXS16; K, grxS14; WT, wild type; OE-S14 O1 and O2, lines overexpressing GRXS14; C-S16 C2 and C4, lines co-suppressed for GRXS16 expression; OE-S16 O4 and O5, lines overexpressing GRXS16. Data in control conditions are means \pm SD of six (rosette weight) and three (chlorophyll) average values originating from independent experiments (at least 5 plants and 5 pigment measurements for each genotype per experiment). In high salt conditions, growth data are means $\pm \mathrm{SD}$ of values gained from seven plants per genotype (the experiment was performed twice and led to similar results) and chlorophyll data are means \pm SD from 5 independent measurements. * ${ }^{* *}$ and $* * *$, significantly different from the WT value with $\mathrm{P}<0.05, \mathrm{P}<$ 0.01 and $\mathrm{P}<0.001$, respectively $(t$-test $)$.

Figure 4. Effect of high salt and prolonged dark exposure on the chlorophyll content of Arabidopsis plants modified in the expression of GRXS14 and/or GRXS16. 
833 (a, b), Chlorophyll content of leaf disks incubated for 2 days on water or $0.15 \mathrm{M} \mathrm{NaCl}$ in the 834 light phytotron conditions $\left(200 \mu \mathrm{mol}\right.$ photons. $\mathrm{m}^{-2} \cdot \mathrm{s}^{-1} ; 8$-h photoperiod) (a) or in the dark (b).

835 Data are means \pm SD of at least eight values gained in two independent experiments (at least 4 836 plants for each genotype per experiment). (c), Chlorophyll content of leaf disks incubated on 837 water in the dark for 3, 4 or 5 days. Means \pm SD of 5 (3 and 4 days) and of 3 (5 days) 838 independent measurements per genotype are presented. (d) Leaves covered with aluminum 839 foil for 8 days on entire 6-week-old plants grown on soil in the light phytotron conditions (8-h 840 photoperiod, $200 \mu$ moles photons $\cdot \mathrm{m}^{-2} \cdot \mathrm{s}^{-1}$ ). (e) Chlorophyll content in leaves covered with 841 aluminum foil for 8 days. Means \pm SD of 11 independent measurements per genotype. KI2, 842 KI1: lines KO for GRXS14 and RNAi for GRXS16; K, grxS14; WT, wild type; O1 and O2, 843 lines overexpressing GRXS14. *,** and ***, significantly different from the WT value with $\mathrm{P}$ $\mathrm{s}<0.05,<0.01$ and $<0.001$, respectively $(t$-test).

Figure 5. Redox status of GRXS14 in leaves of plants grown in control conditions or exposed to environmental constraints.

(a, b) Western analysis of GRXS14 abundance in soluble leaf proteins from control K, WT and $\mathrm{O} 1$ plants extracted in non-reducing conditions either non-alkylated (a) or alkylated using mPEG-maleimide-2000 (b). (c, d, e) Western analysis of GRXS14 redox status in soluble leaf proteins from plants exposed to salt (c, d) or high light (d), or from dark-exposed leaves (e) extracted in non-reducing conditions and alkylated using mPEG-maleimide-2000. (f) Proportion of the $18-\mathrm{kDa}$ GRXS14 form (i.e. completely reduced form) in extracts from WT plants grown in control, high salt and high light conditions and from WT leaves exposed to 855 dark. Control: plants grown under $200 \mu$ moles photons. $\mathrm{m}^{-2} . \mathrm{s}^{-1}$ (8-h photoperiod) for 6 weeks; Salt: plants watered with $0.15 \mathrm{M} \mathrm{NaCl}$ for 1 month; High light: plants grown under 450 $857 \mu$ moles photons. $\mathrm{m}^{-2} \cdot \mathrm{s}^{-1}$ and a long photoperiod $(16 \mathrm{~h})$ for 3 weeks. Dark, leaves exposed to 
858 dark on whole plants in control light conditions for 8 days. Means \pm SD of 15 (control), 10 859 (salt), 3 (high light) and 6 (dark) independent experiments are presented. W, wild-type; K, 860 grxs 14; O1, line overexpressing GRXS14. NS, non-specific band. * and ***, significantly 861 different from the control value with $\mathrm{P}<0.05$ and $<0.001$, respectively $(t$-test).

862

Figure 6. Effect of exposure to dark on the abundance of various plastidial thiol reductases and proteins participating in Fe-S cluster biogenesis.

(a) Western analysis of the abundance of RubisCO large subunit, plastidial thiol reductases (GRXS16, GRXS14, 2-Cys PRX, 2-Cys PRX Ox, PRXQ, PRXIIE and MSRA4 either reduced (Red) or oxidized (Ox)) and various plastidial proteins involved in the maturation of Fe-S proteins (NFU2, NFU3, BOLA4, SUFA1, SUFB, SUFE1 and NFS2). b) Histograms showing the abundance or redox status of several proteins shown in (a). Light, control leaves; Dark, leaves covered with aluminum foil for 8 days on entire 6-week-old plants grown on soil 871 in control phytotron conditions (8-h photoperiod, $200 \mu$ moles photons. $\mathrm{m}^{-2} \cdot \mathrm{s}^{-1} ; 22^{\circ} \mathrm{C}$ ). Leaf 872 proteins were separated using SDS-PAGE in the presence of reductant. Band intensity was 873 quantified using the "Odyssey" software (A.U. Arbitrary Unit). Means \pm SD from 6 874 independent protein extracts are presented. $*$, ** and ***, significantly different from the 875 control value with $\mathrm{P}<0.05, \mathrm{P}<0.01$ and $\mathrm{P}<0.001$, respectively ( $t$-test). 


\section{Supporting information}

Table S1. List of primers used for PCR analysis and cloning.

Table S2. List and characteristics of antibodies used for Western blot analyses.

\section{Figure S1. Characterization of Arabidopsis lines modified in the expression of GRXS16.} PCR analysis of genomic DNA (a) and Western analysis of GRXS14 and GRXS16 abundance (b) in WT and four independent lines carrying the GRXS16 cDNA under the CaMV-35S promoter. C-S16 C2 and C4, lines co-suppressed for GRXS16 expression; OE-S16 O4 and O5, lines overexpressing GRXS16.

Figure S2. Phenotype of Arabidopsis plants knockout for GRXS14, knockdown for GRXS16 and restored with GRXS14 expression.

Two independent KI1 lines restored for GRXS14 expression, termed KI1-R1and KI1-R2, were generated following transformation to integrate the GRXS14 cDNA under the CaMV-35S promoter in genomic DNA. (a) Western analysis of RubisCO and GRXS14 abundance in leaves of 6-week-old plants. (b) Rosette weight of plants grown for 6 weeks in control conditions, moderate light $\left(200 \mu \mathrm{mol}\right.$ photons.m $\left.\mathrm{m}^{-2} \cdot \mathrm{s}^{-1}\right)$ and short photoperiod $(8 \mathrm{~h})$ at $22^{\circ} \mathrm{C}$. Data are means \pm SD of ten to fifteen independent measurements per genotype. (c) Chlorophyll content in leaves covered with aluminum foil for 8 days. Means \pm SD of 13 (WT), 3 (K and KI1) and 5 (KI1-R1 and KI1-R2) independent measurements. KI1: line KO for GRXS14 and RNAi for GRXS16; K, grxS14; WT, wild type; KI1-R1 and KI1-R2: lines KO for GRXS14, RNAi for GRXS16 and restored for GRXS14 expression. *, significantly different from the WT value with $\mathrm{P}<0.05$ ( $t$-test). 
900

901

902

903

904

905

906

907

908

909

910

911

912

913

914

915

916

917

918

919

920

921

922

923

924

Figure S3. Maximal photochemical efficiency of photosystem II $\left(F_{v} / F_{m}\right)$ in plants modified for the expression of GRXS14 and/or GRXS16 in various environmental conditions.

(a) Plants grown for 6 weeks in control light conditions $\left(200 \mu \mathrm{mol}\right.$ photons. $\mathrm{m}^{-2} . \mathrm{s}^{-1}$; 8 -h photoperiod) at $22^{\circ} \mathrm{C}$. (b), Plants aged of 21 days grown in control conditions and then watered with $0.15 \mathrm{M} \mathrm{NaCl}$ for 31 days in the same light conditions. (c) Leaf disks incubated on water, $0.15 \mathrm{M} \mathrm{NaCl}, 0.15 \mathrm{M} \mathrm{KCl}$ or $0.3 \mathrm{M}$ mannitol in control light conditions for $48 \mathrm{~h}$. (d) Leaf disks incubated on water or $0.15 \mathrm{M} \mathrm{NaCl}$ in the dark for $48 \mathrm{~h}$. Data in (a, b) are means \pm SD of at least five average values originating from independent plants (at least 5 measurements per plant). Data in (c, Water and $\mathrm{NaCl})$ are means $\pm \mathrm{SD}$ of six average values originating from independent experiments (10 measurements per genotype per experiment) and in (c, $\mathrm{KCl}$, mannitol) and (d), means $\pm \mathrm{SD}$ of at least eight values gained in two independent experiments (at least 4 plants for each genotype per experiment). KI2, KI1: lines KO for GRXS14 and RNAi for GRXS16; K, grxS14; WT, wild type; OE-S14 O1 and O2, lines overexpressing *,** and ***, significantly different from the WT value with $\mathrm{P}<0.05, \mathrm{P}$ $<0.01$ and $\mathrm{P}<0.001$, respectively (t-test). ( $t$-test).

Figure S4. Characteristics related to plastidial Fe-S proteins in plants modified in the expression of GRXS14 and/or GRXS16.

Ratio of the heights of the fluorescence peaks emitted in liquid nitrogen by photosystems I and II (a) and Western analysis of the abundance of components of the plastidial Fe-S machinery (b, c, d). Western analysis of RubisCO LSU, 2-Cys PRX, FDX2 (two isoforms), NFU2, SUFE1, NFU3, SUFA1, SUFB and NFS2 abundance (b). Quantification of the abundance of NFU2 (c) and SUFE1 (d) abundance. Data in $(\mathbf{c}, \mathbf{d})$ are means \pm SD from four independent extracts per genotype. KI1 and KI2: lines KO for GRXS14 and RNAi for 
925 GRXS16; K, grxS14; WT, wild type; OE-S14 O1 and O2, lines overexpressing GRXS14; C-

926 S16 C2 and C4, lines co-suppressed for GRXS16 expression; OE-S16 O4 and O5, lines

927 overexpressing GRXS16. Band intensity was quantified using the Odyssey software (A.U., 928 arbitrary unit). *, ** and ***, significantly different from the WT value with $\mathrm{P}<0.05, \mathrm{P}<$ 9290.01 and $\mathrm{P}<0.001$, respectively $(t$-test).

930

Figure S5. Responses to photooxidative conditions of Arabidopsis lines modified in the

932

933

934

935

936

937

938

939

940

941

942

943

944

945

946

947

948 expression of GRXS14 and/or GRXS16.

(a) Growth in moderate photooxidative stress conditions. Plants were grown from sowing for 3 weeks in high light conditions $\left(450 \mu \mathrm{mol}\right.$ photons. $\left.\mathrm{m}^{-2} . \mathrm{s}^{-1}\right)$ and long photoperiod $(16 \mathrm{~h})$ at $22^{\circ} \mathrm{C}$. (a) Rosette weight. (b) Leaf chlorophyll content. (c,d) Response to acute photooxidative stress conditions. (c) Five-week old plants exposed to high light (1400 $\mu$ mol.m $\left.\mathrm{m}^{-2} \mathrm{~s}^{-1}\right)$ at low temperature $\left(6^{\circ} \mathrm{C}\right)$ for 11 days. (d) Maximal photochemical efficiency of photosystem II $\left(\mathrm{F}_{\mathrm{v}} / \mathrm{F}_{\mathrm{m}}\right)$ of leaf disks incubated on $2 \mu \mathrm{M}$ methyl viologen for $48 \mathrm{~h}$ in the phytotron (200 $\mu \mathrm{mol}$ photons. $\mathrm{m}^{-2} \cdot \mathrm{s}^{-1} ; 8$-h photoperiod). KI1 and KI2: lines KO for GRXS14 and RNAi for GRXS16; K, grxS14; WT, wild type; OE-S14 O1 and O2, lines overexpressing GRXS14; C-S16 C2, line co-suppressed for GRXS16 expression; OE-S16 O4, line overexpressing GRXS16. Data are means $\pm \mathrm{SD}$ of six (rosette weight) and three (other data) average values originating from independent experiments (at least 5 plants and 3 measurements for each genotype per experiment). *** and ***, significantly different from the WT value with $\mathrm{P}<0.05, \mathrm{P}<0.01$ and $\mathrm{P}<0.001$, respectively $(t$-test).

Figure S6. Responses of Arabidopsis plants modified in the expression of GRXS16 to osmotic and oxidative constraints. 
949 (a) Effect of $\mathrm{NaCl}$ and methyl viologen (MV) on the PSII activity of leaf discs. The maximal 950 photochemical efficiency of photosystem II $\left(\mathrm{F}_{\mathrm{v}} / \mathrm{F}_{\mathrm{m}}\right)$ was measured on disks incubated on 951 water, $0.15 \mathrm{M} \mathrm{NaCl}$ or $2 \mu \mathrm{M} \mathrm{MV}$ in the phytotron conditions $\left(200 \mu\right.$ mol photons. $\mathrm{m}^{-2} \cdot \mathrm{s}^{-1} ; 8-\mathrm{h}$ 952 photoperiod; $22^{\circ} \mathrm{C}$ ) for $48 \mathrm{~h}$. Mean \pm SD of 10 independent measurements. (b-e) Growth and 953 photosynthetic parameters of plants exposed to $\mathrm{NaCl}$. Plants aged of 21 days grown in control 954 conditions were then watered with $0.15 \mathrm{M} \mathrm{NaCl}$ in the phytotron conditions for 31 days (b) 955 Rosette weight of salt-treated plants. Mean \pm SD of 5 independent values. (c) Maximal 956 photochemical efficiency of photosystem II $\left(\mathrm{F}_{\mathrm{v}} / \mathrm{F}_{\mathrm{m}}\right)$ of salt-treated plants. Mean \pm SD of 20 957 independent measurements performed on 4 plants. (d) Leaf chlorophyll content of salt-treated 958 plants. Mean \pm SD of 5 independent measurements. C-S16 C2 and C4, lines co-suppressed for 959 GRXS16 expression; WT, wild type; OE-S16 O4 and O5, lines overexpressing GRXS16. * and 960 ***, significantly different from the WT value with $\mathrm{P}<0.05$ and $<0.001$, respectively $(t$-test).

Figure S7. Effect of exposure to dark on the chlorophyll content of Arabidopsis plants modified for GRXS14 and/or GRXS16 expression.

964 (a, b) Leaf disks incubated on water in the dark for 4 days. (c) Chlorophyll content of disks 965 incubated in the dark for 4 days. Means \pm SD of 6 independent values per genotype. (d) Leaves covered with aluminum foil for 8 days on entire 6-week old plants grown on soil in standard light conditions (8-h photoperiod, $200 \mu$ moles photons.m $\mathrm{m}^{-2} \cdot \mathrm{s}^{-1} ; 22^{\circ} \mathrm{C}$ ). (e) Chlorophyll content in leaves covered with aluminum foil for 8 days. Mean \pm SD of 6 independent measurements per genotype. KI2, KI1: lines KO for GRXS14 and RNAi for GRXS16; K, grxS14; WT, wild type; OE-S14 O1 and O2, lines overexpressing GRXS14; C-

971 S16 C2 and C4, lines co-suppressed for GRXS16 expression; OE-S16 O4 and O5, lines 972 overexpressing GRXS16. 
Figure 1

(a)
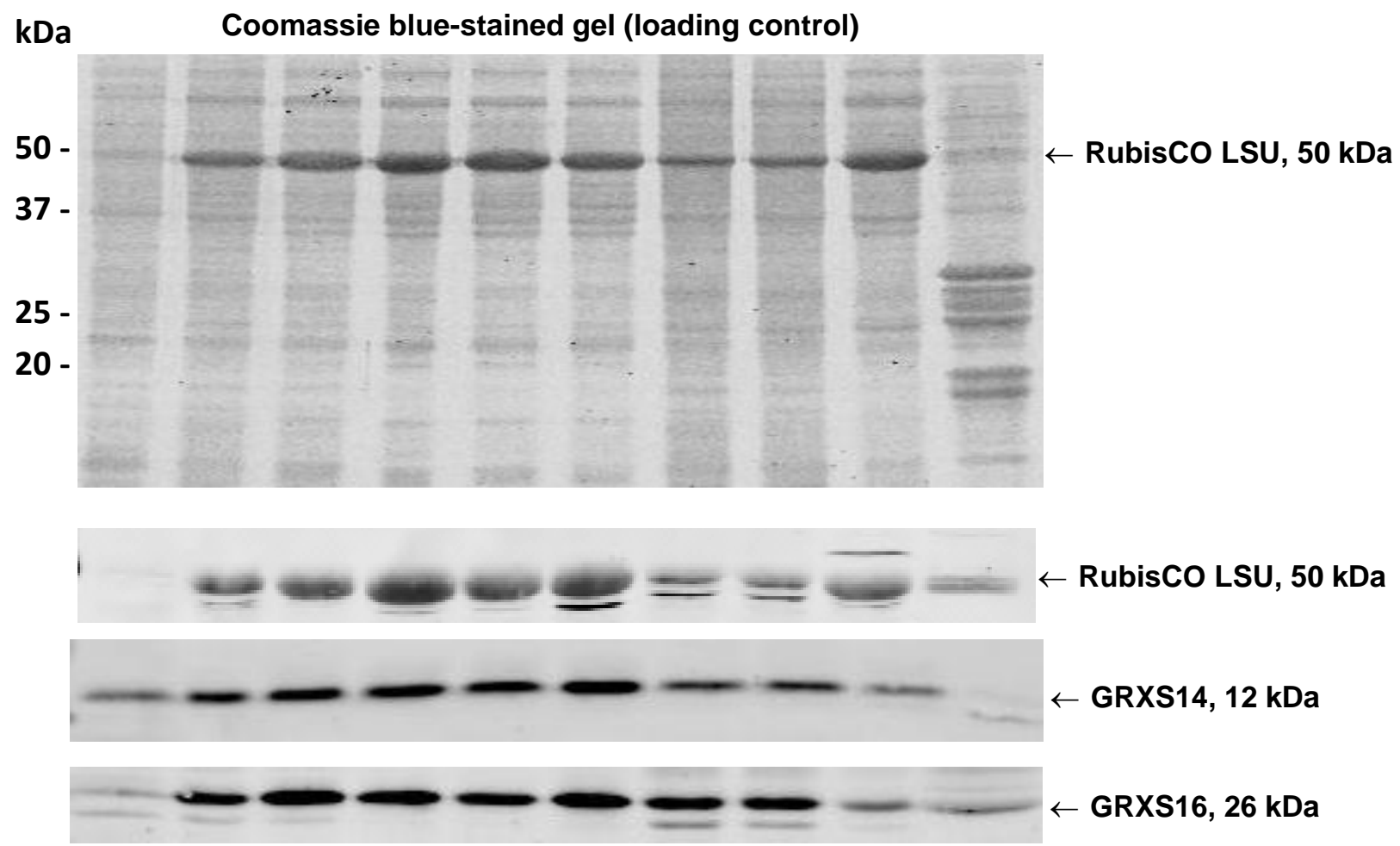

$\begin{array}{llllllllll}R & \text { St } & \text { YL } & \text { AL } & \text { OL } & \text { CL } & \text { FB } & \text { F } & \text { GP } & \text { Se }\end{array}$

(b)

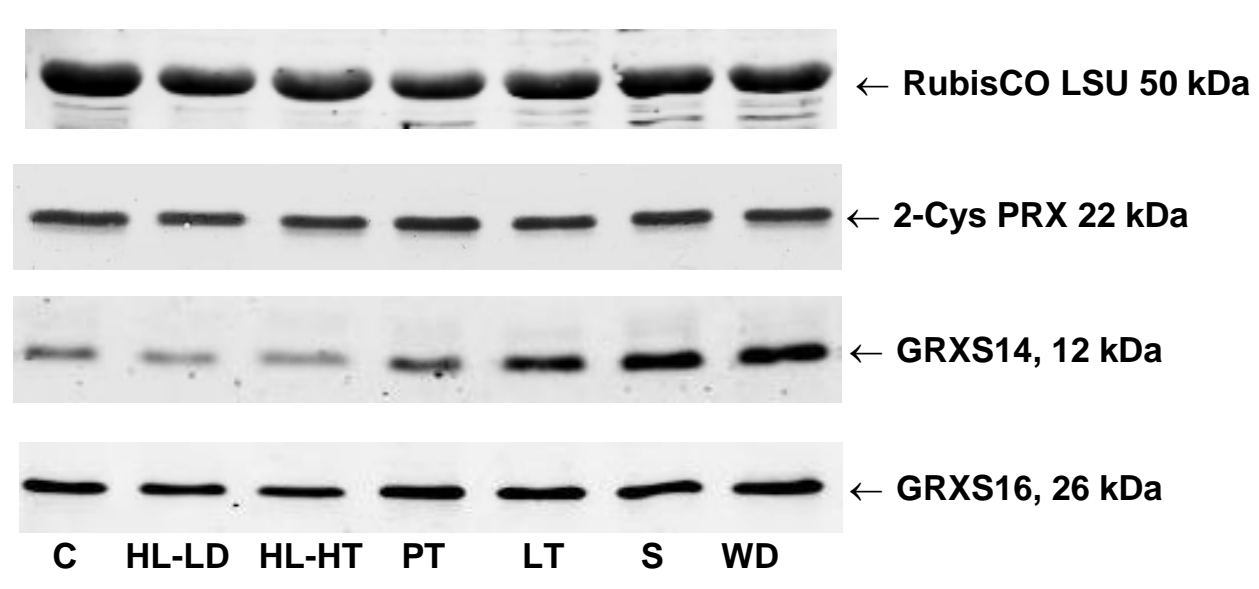


Figure 2

(a)

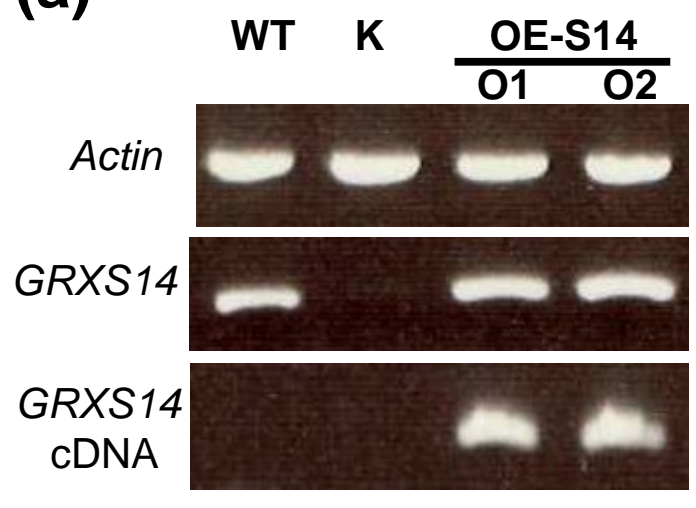

RNAi construct

(b)

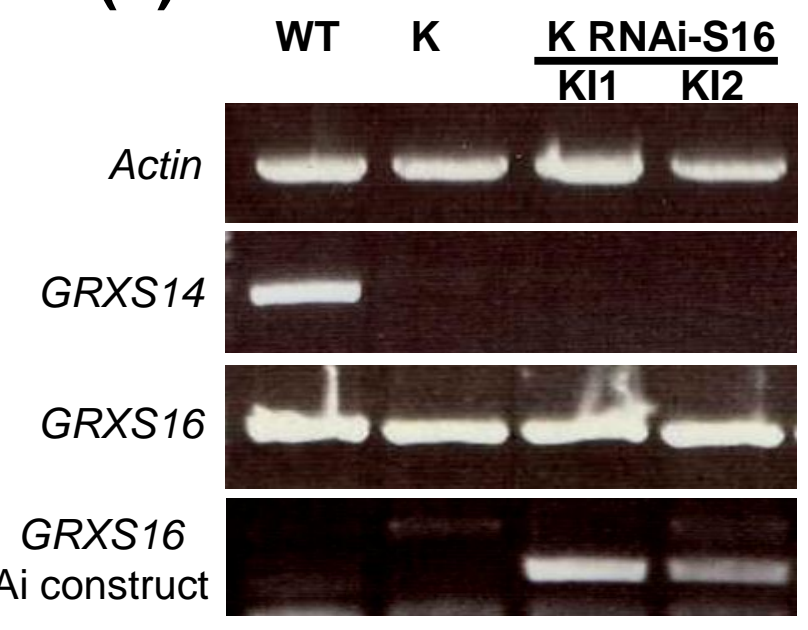

(c)

WT $K \quad \frac{\text { OE-S14 }}{01 \quad 02}$

GRXS14

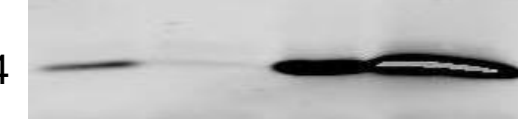

GRXS16 (d) WT K $\frac{\text { KRNAi-S16 }}{\mathrm{KI1} \quad \mathrm{KI} 2}$

GRXS14

GRXS16

$\leftarrow 26 \mathrm{kDa}$ 
(a)

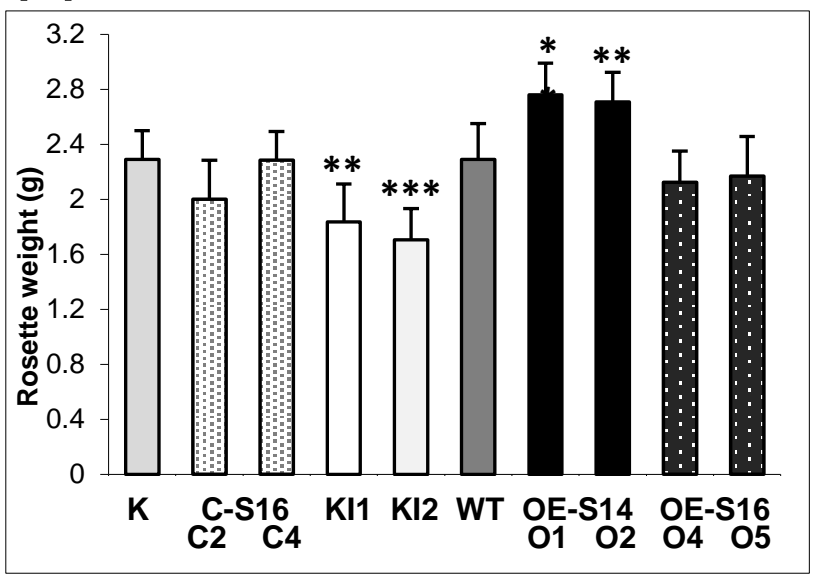

(b)

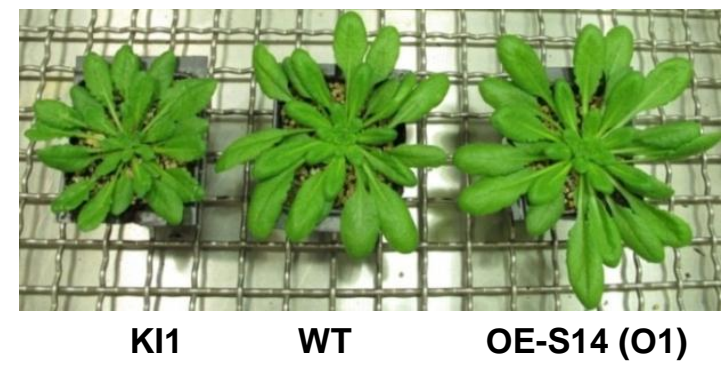

(c)

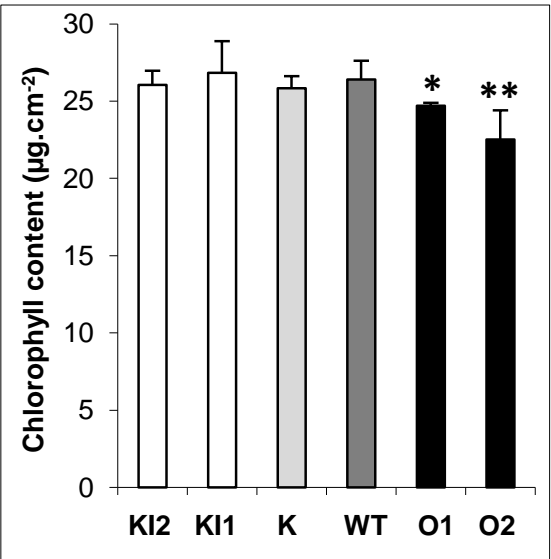

(d) High salt

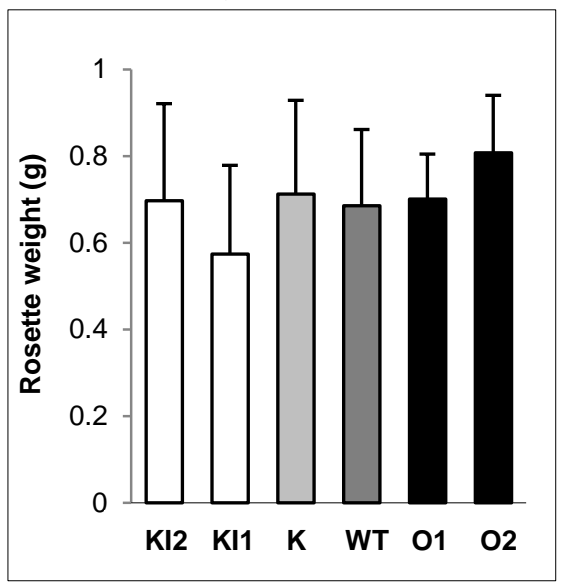

(e)

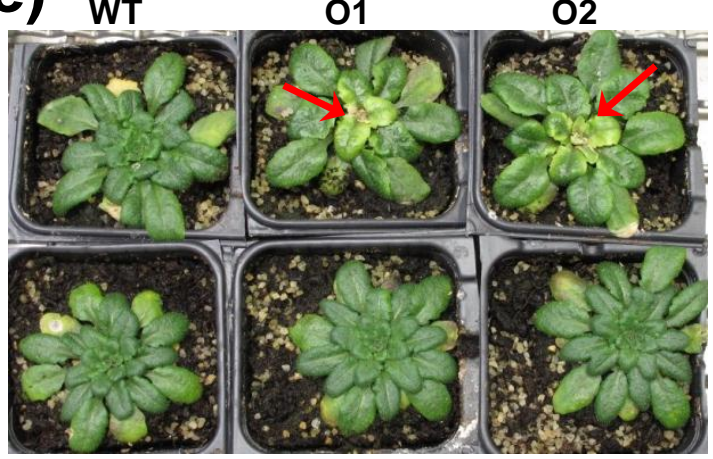

K

KI1

$\mathrm{K} / 2$

(f)

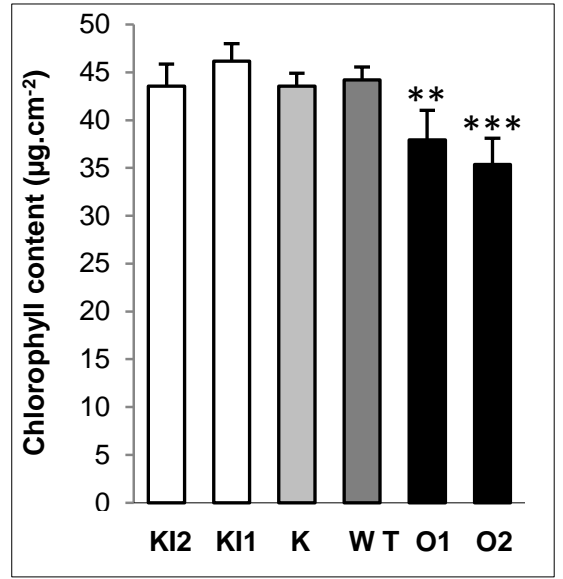




\section{Pageingerre 4}

(a)

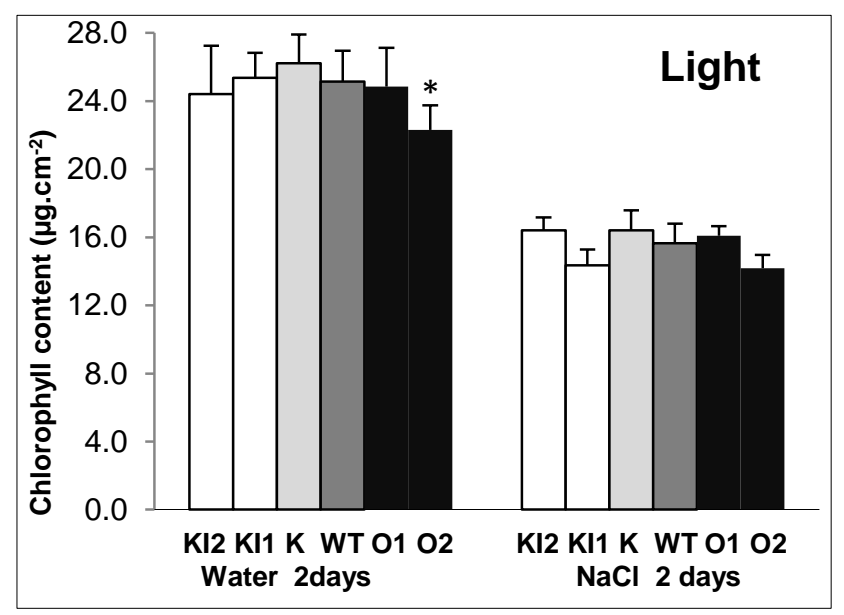

(b)

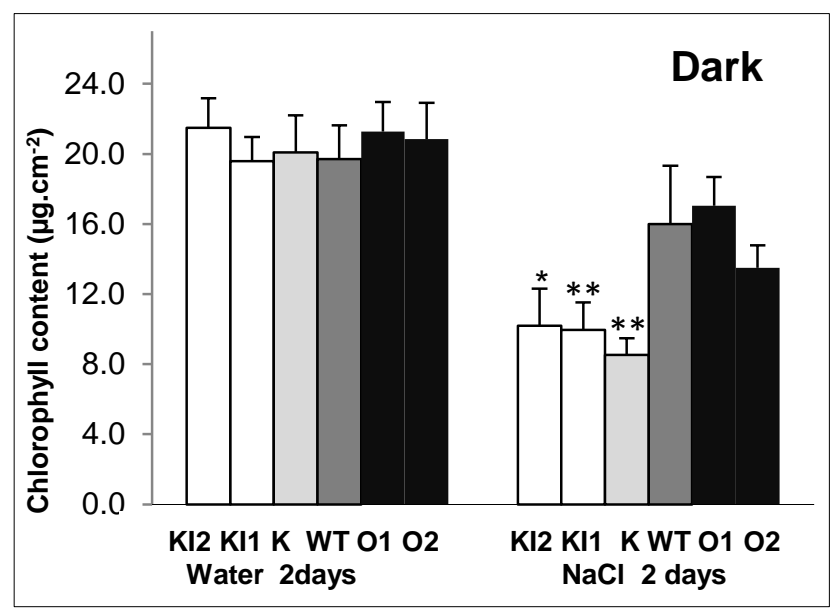

(c)

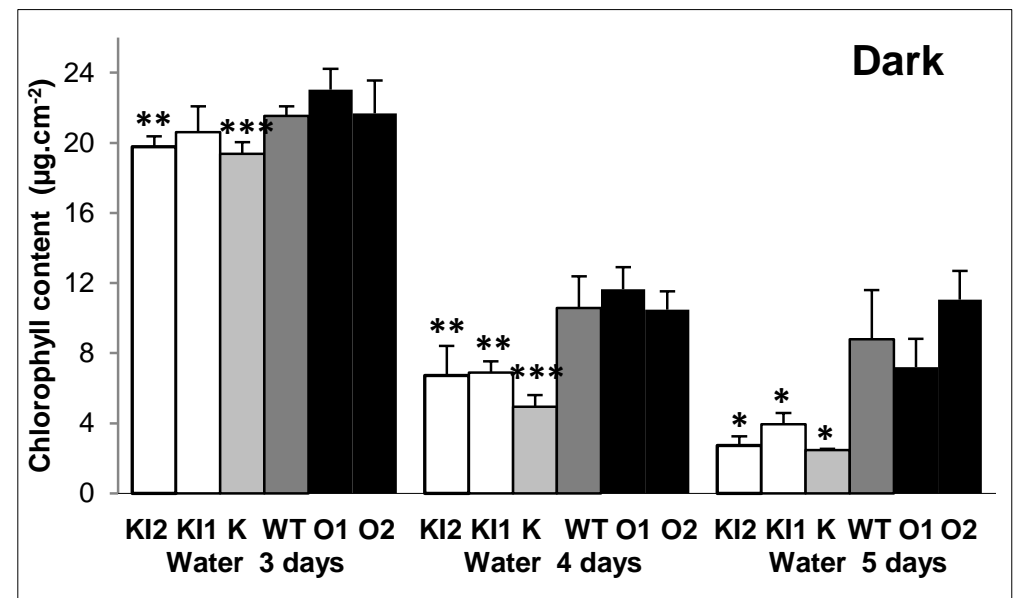

(d)

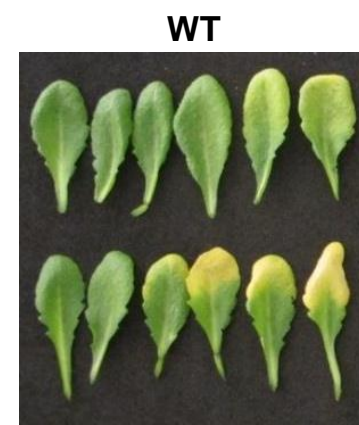

K
01

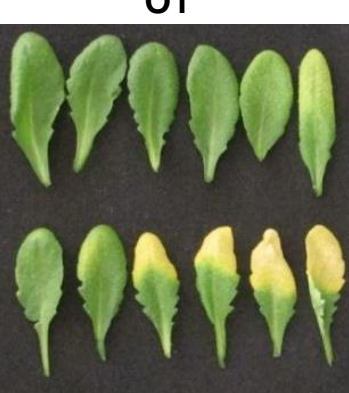

KI1
02

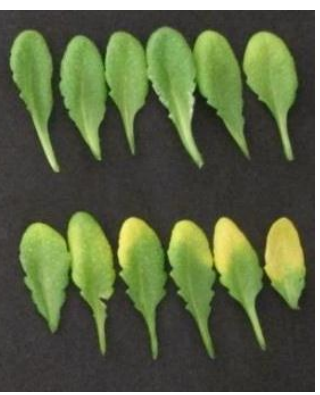

KI2 (e)

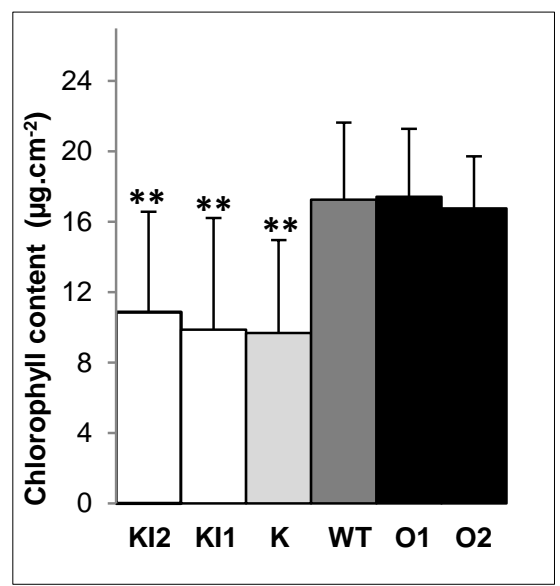


Figure 5

(a)

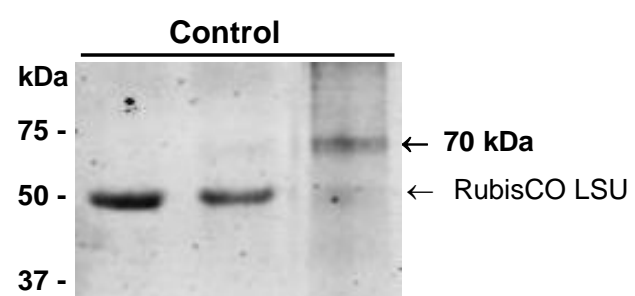

25 . $\quad \leftarrow 24 \mathrm{kDa} \quad$ (d)

20 -

$15-$

10 -

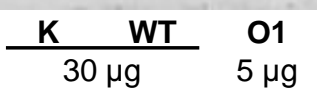

(b)

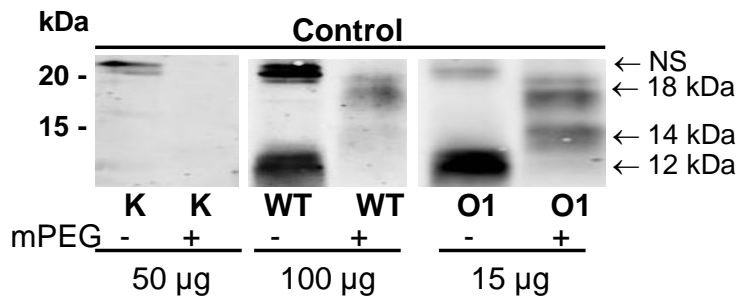

(c)
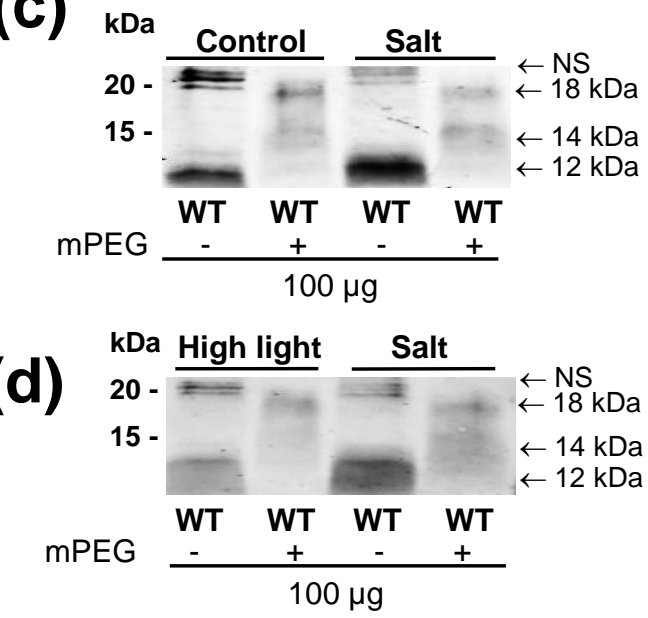

(e)

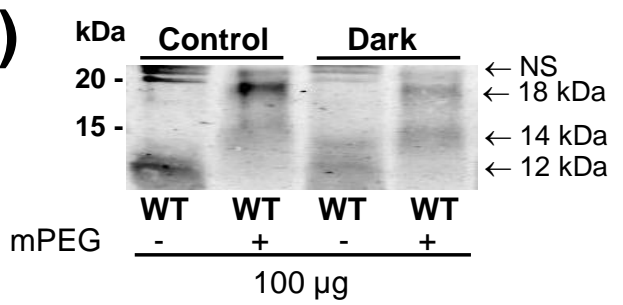

(f)

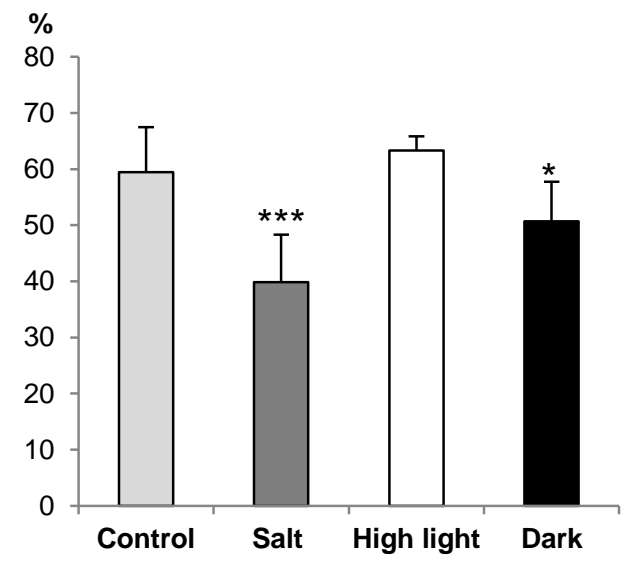


Figure 6

(a)
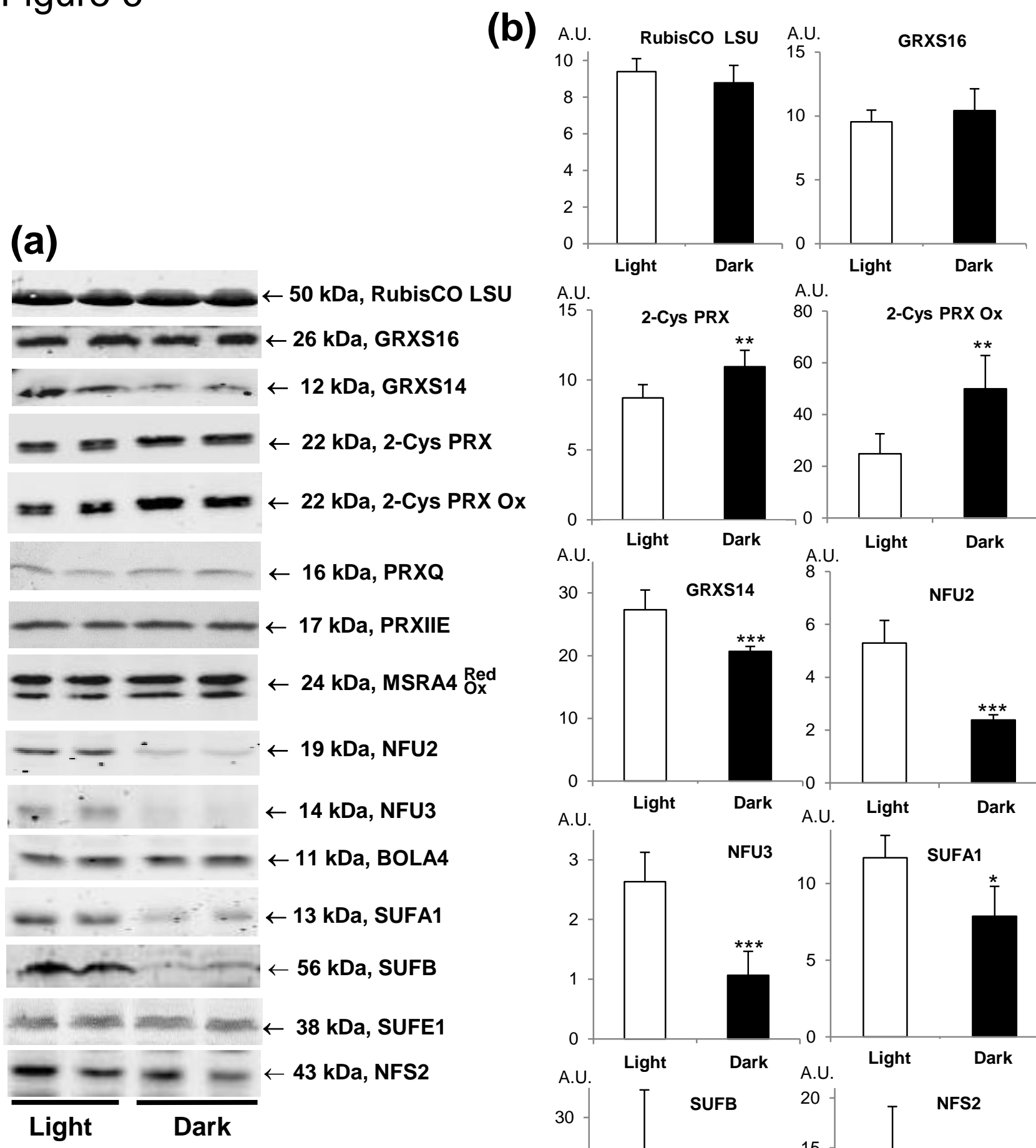

A.U.

80 2-Cys PRX Ox
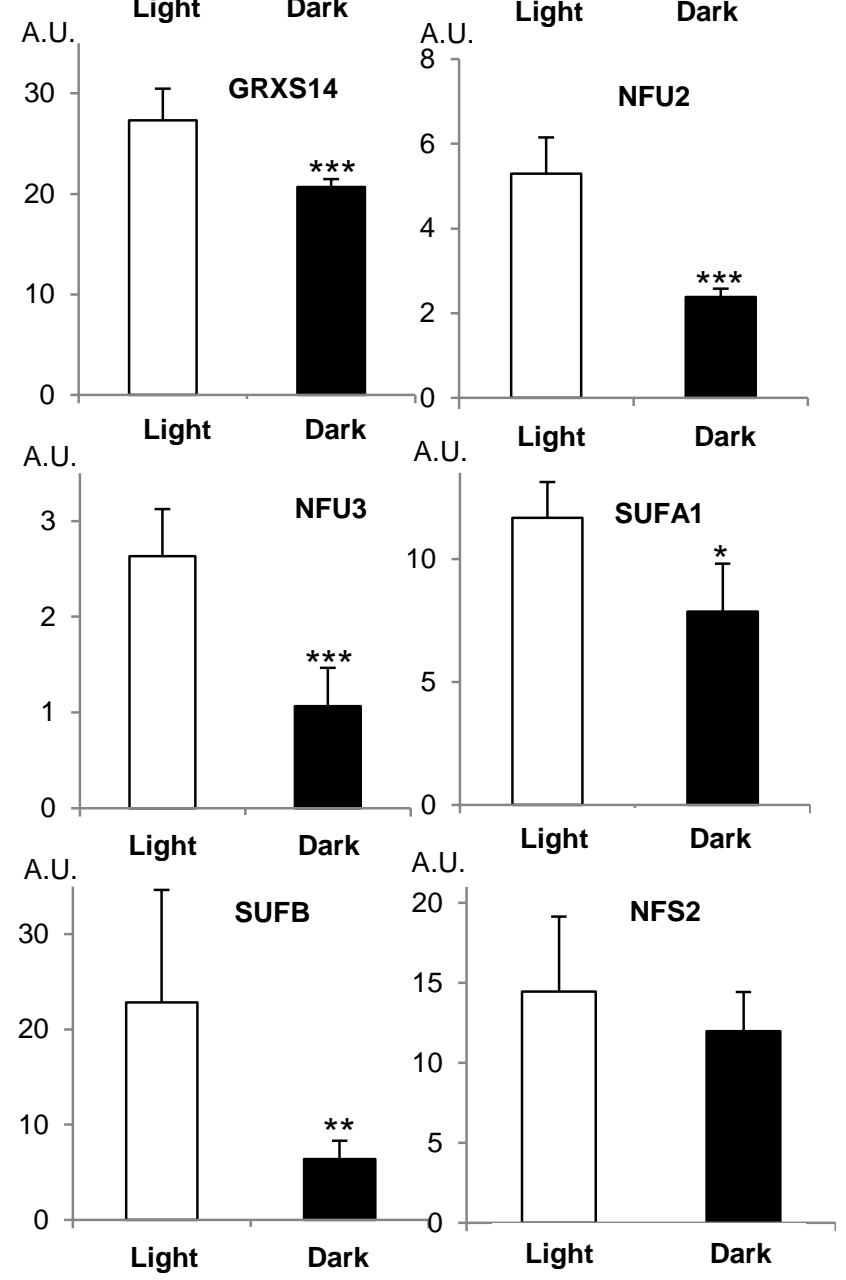

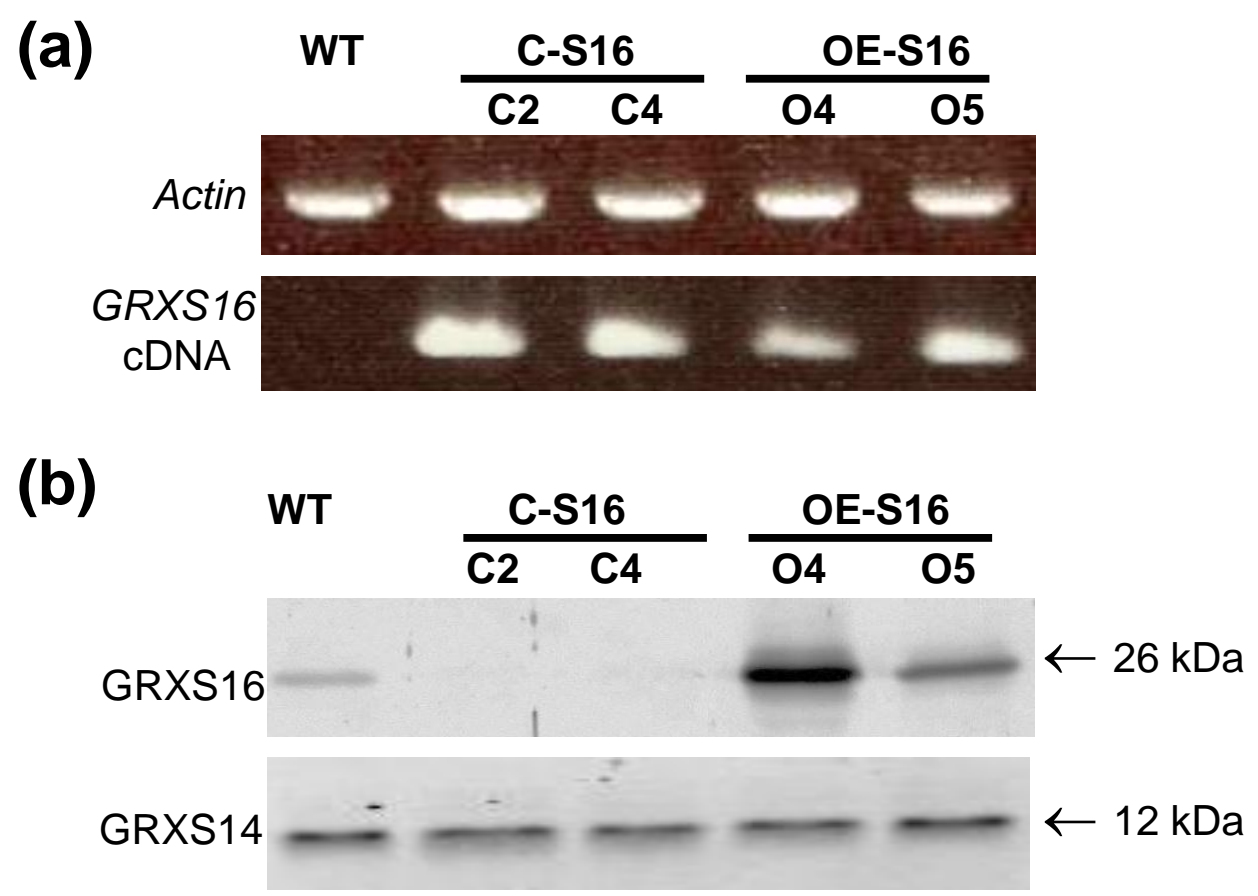

Figure S1. Characterization of Arabidopsis lines modified in the expression of GRXS16.

PCR analysis of genomic DNA (a) and Western analysis of GRXS14 and GRXS16 abundance (b) in WT and four independent lines carrying the GRXS16 cDNA under the CaMV-35S promoter. C-S16 C2 and C4, lines co-suppressed for GRXS16 expression; OE-S16 O4 and O5, lines overexpressing GRXS16. 


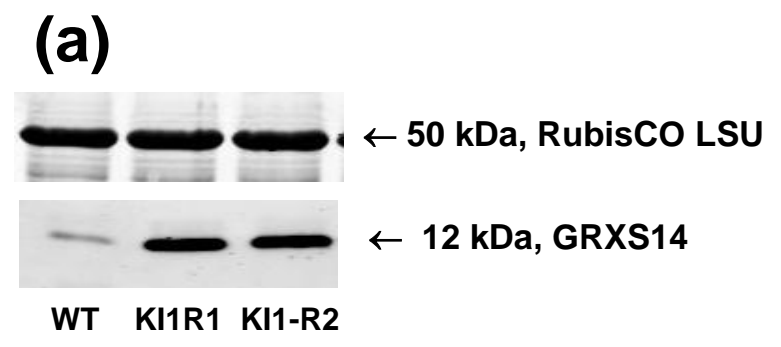

(b)

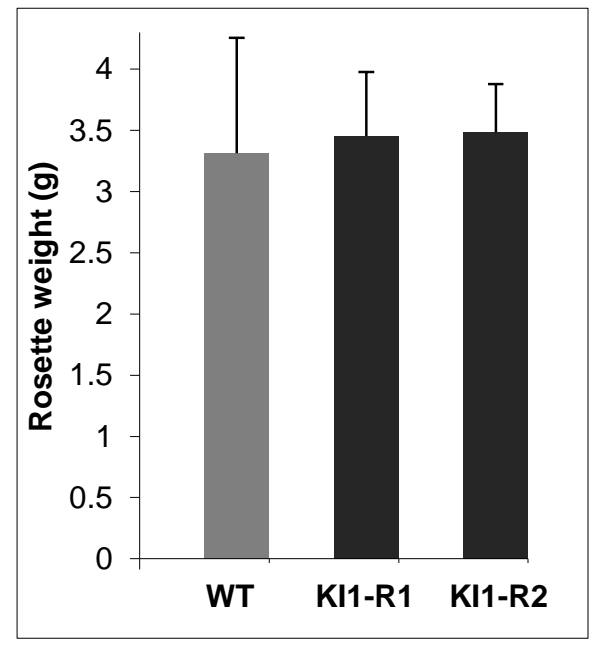

(c)

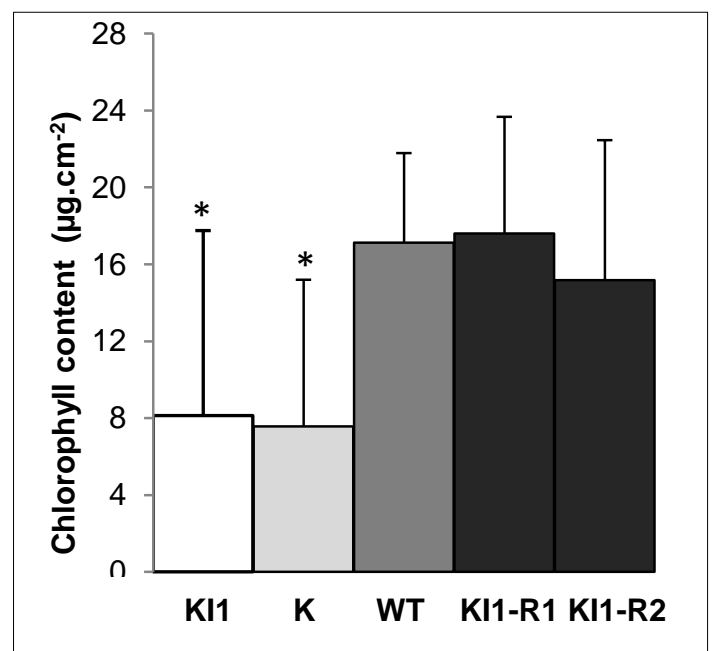

Figure S2. Phenotype of Arabidopsis plants knockout for GRX14, knockdown for GRX16 and restored with GRXS14 expression.

Two independent KI1 lines restored for GRXS14 expression, termed KI1-R1and KI1-R2, were generated following transformation to integrate the GRXS14 cDNA under the CaMV-35S promoter in genomic DNA. (a) Western analysis of RubisCO and GRXS14 abundance in leaves of 6-weekold plants. (b) Rosette weight of plants grown for 6 weeks in control conditions, moderate light $\left(200 \mu \mathrm{mol}\right.$ photons. $\left.\mathrm{m}^{-2} \cdot \mathrm{s}^{-1}\right)$ and short photoperiod $(8 \mathrm{~h})$ at $22^{\circ} \mathrm{C}$. Data are means $\pm \mathrm{SD}$ of ten to fifteen independent measurements per genotype. (c) Chlorophyll content in leaves covered with aluminum foil for 8 days. Means \pm SD of 13 (WT), 3 (K and KI1) and 5 (KI1-R1 and KI1-R2) independent measurements. KI1: line KO for GRXS14 and RNAi for GRXS16; K, grxS14; WT, wild type; KI1-R1 and KI1-R2 lines: KO for GRXS14, RNAi for GRXS16 and restored for GRXS14 expression. *, significantly different from the WT value with $\mathrm{P}<0.05$ ( $t$-test). 


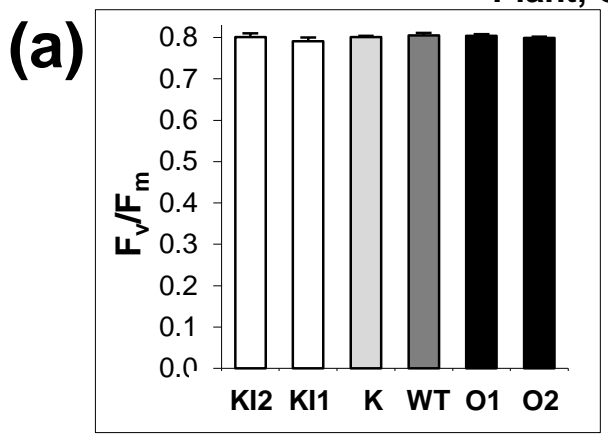

Control plants

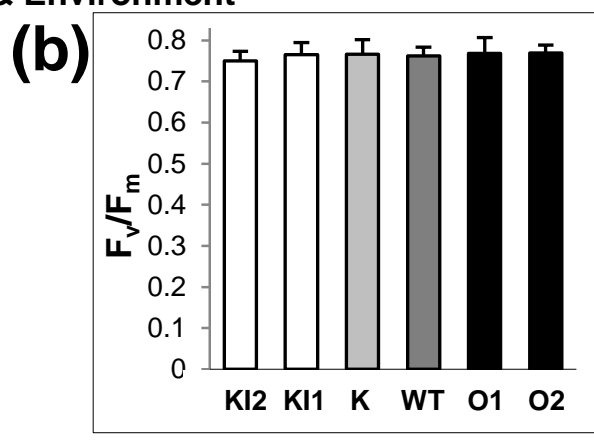

Plants exposed to high salt

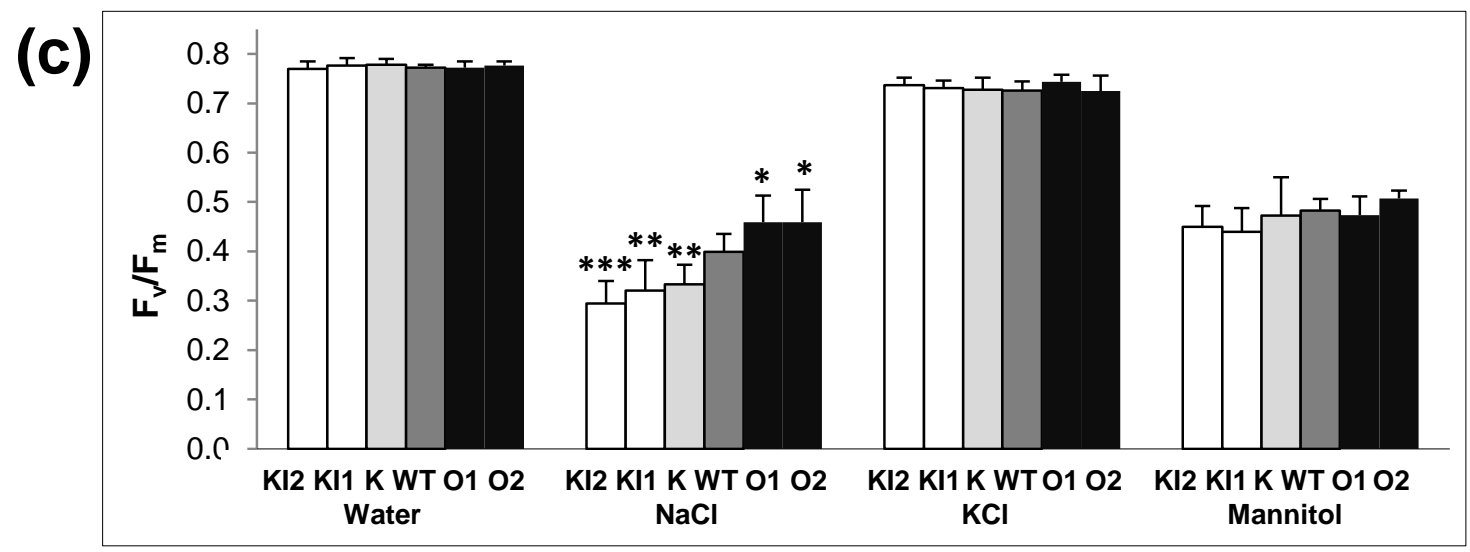

Leaf disks in the light for $48 \mathrm{~h}$

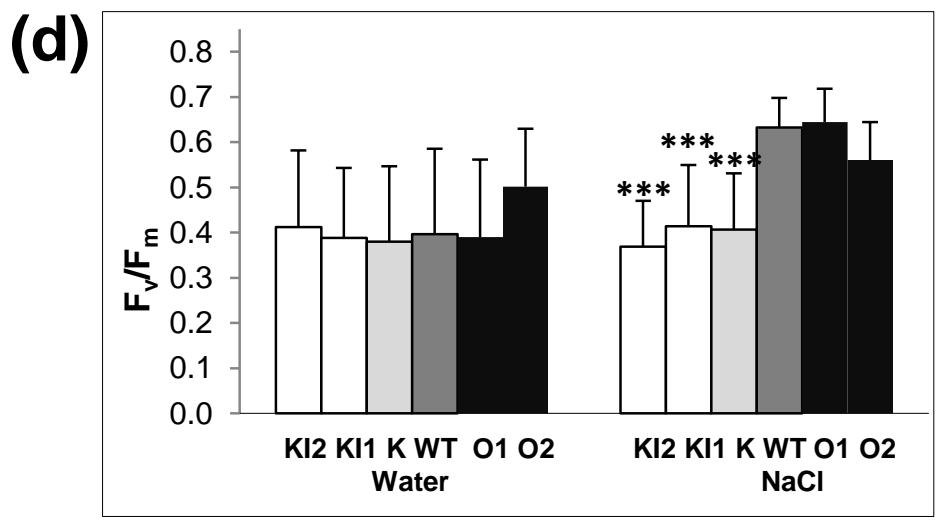

Leaf disks in the dark for $48 \mathrm{~h}$

Figure S3. Maximal photochemical efficiency of photosystem II $\left(F_{v} / F_{m}\right)$ in plants modified for the expression of GRXS14 and/or GRXS16 in various environmental conditions.

(a) Plants grown for 6 weeks in control light conditions $\left(200 \mu\right.$ mol photons. $\mathrm{m}^{-2} \cdot \mathrm{s}^{-1} ; 8$-h photoperiod) at $22^{\circ} \mathrm{C}$. (b), Plants aged of 21 days grown in control conditions and then watered with $0.15 \mathrm{M} \mathrm{NaCl}$ for 31 days in the same light conditions. (c) Leaf disks incubated on water, $0.15 \mathrm{M} \mathrm{NaCl}, 0.15 \mathrm{M} \mathrm{KCl}$ or $0.3 \mathrm{M}$ mannitol in control light conditions for $48 \mathrm{~h}$. (d) Leaf disks incubated on water or $0.15 \mathrm{M} \mathrm{NaCl}$ in the dark for $48 \mathrm{~h}$. Data in (a, b) are means \pm SD of at least five average values originating from independent plants (at least 5 measurements per plant). Data in (c, Water and $\mathrm{NaCl}$ ) are means $\pm \mathrm{SD}$ of six average values originating from independent experiments (10 measurements per genotype per experiment) and in (c, $\mathrm{KCl}$, mannitol) and (d), means $\pm \mathrm{SD}$ of at least eight values gained in two independent experiments (at least 4 plants for each genotype per experiment). KI2, KI1: lines KO for GRXS14 and RNAi for GRXS16; K, grxS14; WT, wild type; OE-S14 O1 and O2, lines overexpressing *,** and ***, significantly different from the WT value with $\mathrm{P}<0.05, \mathrm{P}<0.01$ and $\mathrm{P}<0.001$, respectively (t-test). $(t$-test). 
(a)

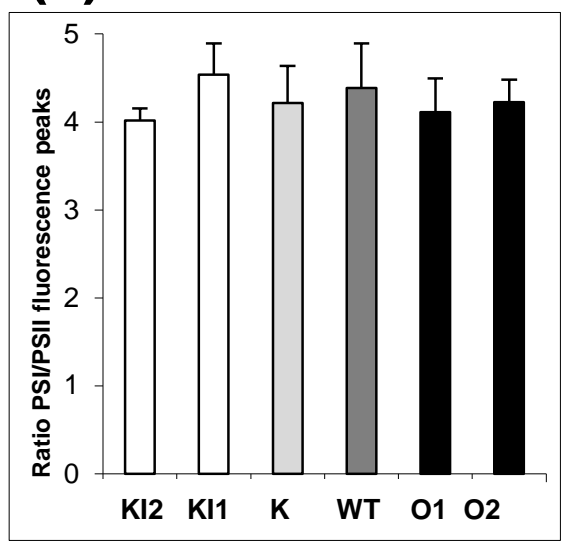

أر $\leftarrow 50$ kDa, RubisCo LSU

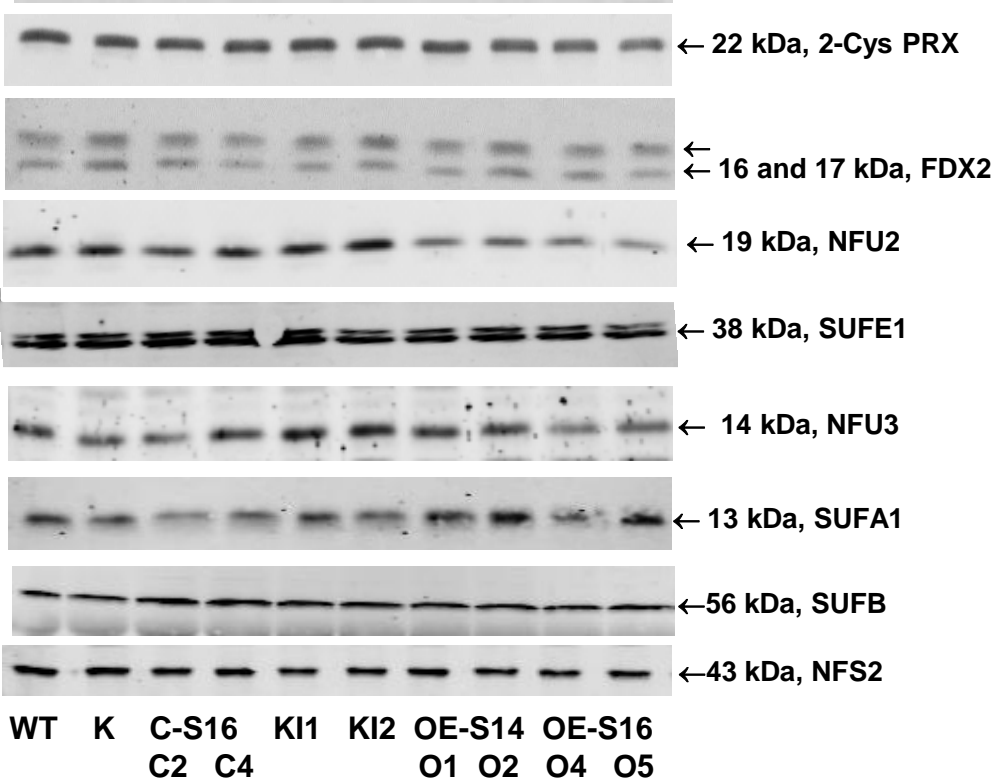

(c)

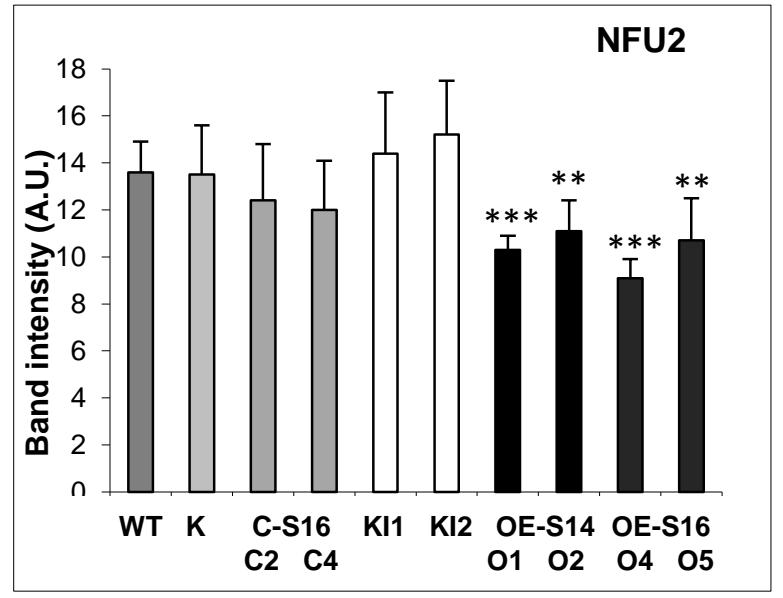

(d)

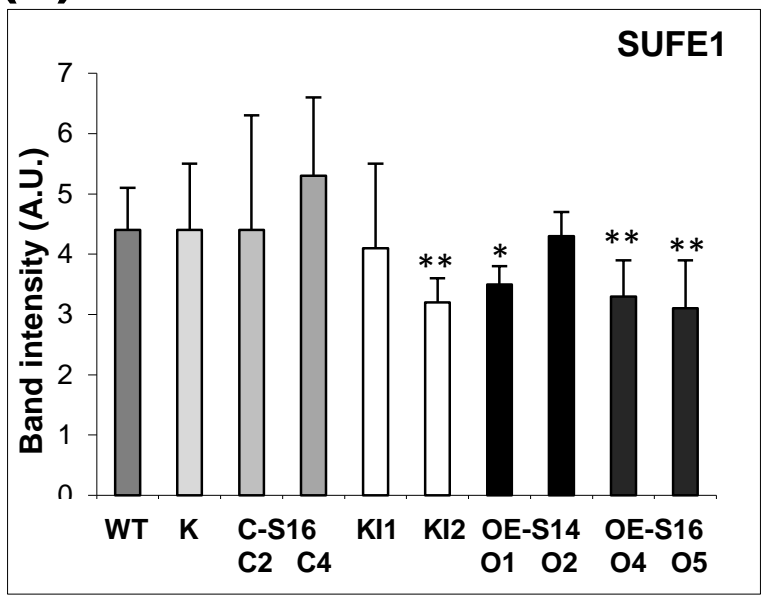

Figure S4. Characteristics related to plastidial Fe-S proteins in plants modified in the expression of GRXS14 and/or GRXS16.

Ratio of the heights of the fluorescence peaks emitted in liquid nitrogen by photosystems I and II (a) and Western analysis of the abundance of components of the plastidial Fe-S machinery (b, c, d).

Western analysis of RubisCO LSU, 2-Cys PRX, FDX2 (two isoforms), NFU2, SUFE1, NFU3, SUFA1, SUFB and NFS2 abundance (b). Quantification of the abundance of NFU2 (c) and SUFE1 (d) abundance. Data in (c, d) are means \pm SD from four independent extracts per genotype. KI1 and KI2: lines KO for GRXS14 and RNAi for GRXS16; K, grxS14; WT, wild type; OE-S14 O1 and O2, lines overexpressing GRXS14; C-S16 C2 and C4, lines co-suppressed for GRXS16 expression; OE-S16 O4 and O5, lines overexpressing GRXS16. Band intensity was quantified using the Odyssey software (A.U., arbitrary unit). *, $*^{* *}$ and ${ }^{* * *}$, significantly different from the WT value with $\mathrm{P}<0.05, \mathrm{P}<0.01$ and $\mathrm{P}<0.001$, respectively $(t-$ test). 
(a)

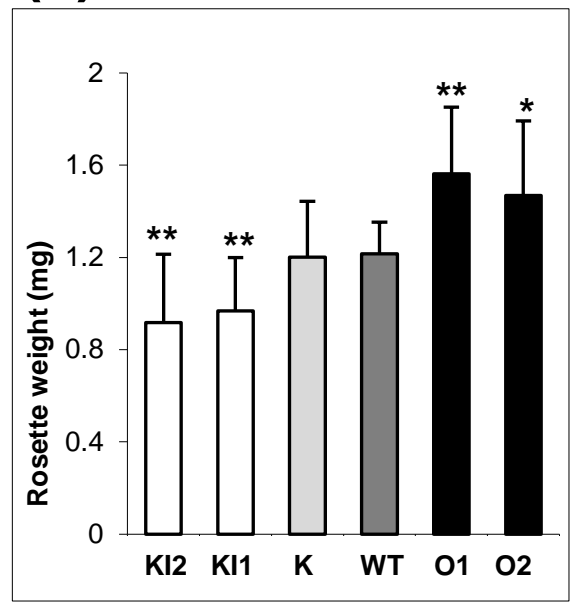

(c)

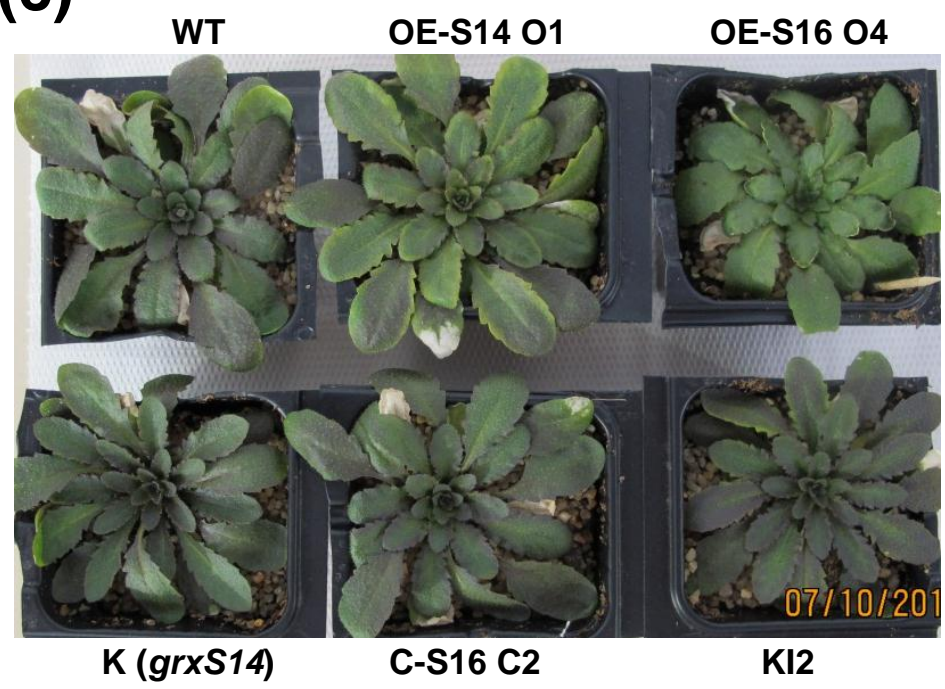

(b)

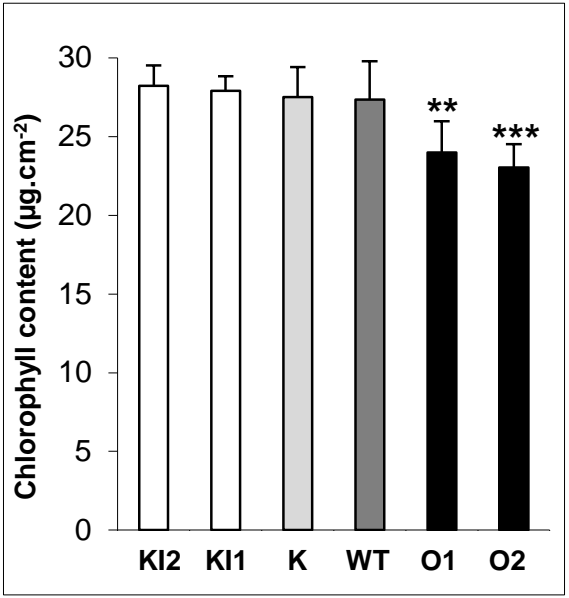

(d)

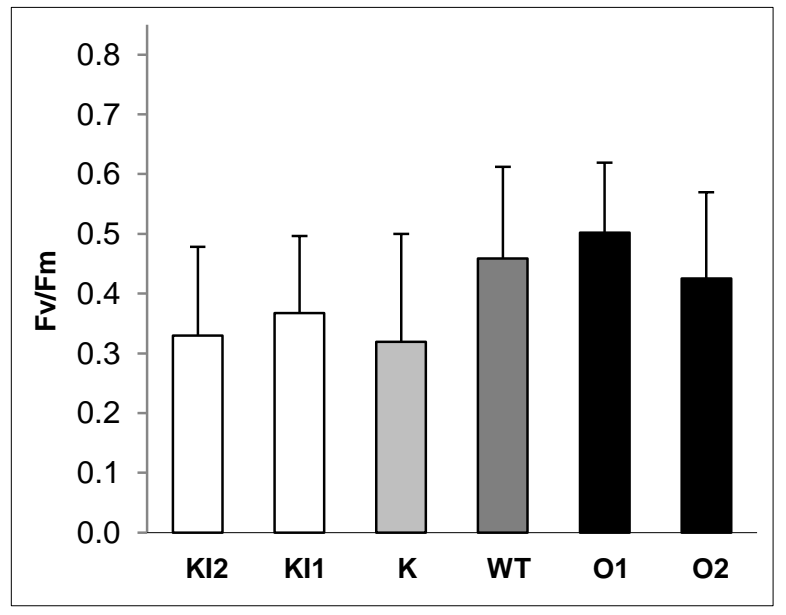

Figure S5. Responses to photooxidative conditions of Arabidopsis lines modified in the expression of GRXS14 and/or GRXS16.

(a) Growth in moderate photooxidative stress conditions. Plants were grown from sowing for 3 weeks in high light conditions $\left(450 \mu \mathrm{mol}\right.$ photons. $\left.\mathrm{m}^{-2} \cdot \mathrm{s}^{-1}\right)$ and long photoperiod $(16 \mathrm{~h})$ at $22^{\circ} \mathrm{C}$. (a) Rosette weight. (b) Leaf chlorophyll content. (c,d) Response to acute photooxidative stress conditions. (c) Five-week old plants exposed to high light $\left(1400 \mu \mathrm{mol} . \mathrm{m}^{-2} . \mathrm{s}^{-1}\right)$ at low temperature $\left(6^{\circ} \mathrm{C}\right)$ for 11 days. (d) Maximal photochemical efficiency of photosystem II $\left(\mathrm{F}_{\mathrm{v}} / \mathrm{F}_{\mathrm{m}}\right)$ of leaf disks incubated on $2 \mu \mathrm{M}$ methyl viologen for $48 \mathrm{~h}$ in the phytotron $(200 \mu \mathrm{mol}$ photons. $\mathrm{m}^{-2} \cdot \mathrm{s}^{-1}$; 8-h photoperiod). KI1 and KI2: lines KO for GRXS14 and RNAi for GRXS16; K, grxS14; WT, wild type; OE-S14 O1 and O2, lines overexpressing GRXS14; C-S16 C2, line co-suppressed for GRXS16 expression; OE-S16 O4, line overexpressing GRXS16. Data are means \pm SD of six (rosette weight) and three (other data) average values originating from independent experiments (at least 5 plants and 3 measurements for each genotype per experiment). *, ** and $* * *$, significantly different from the WT value with $\mathrm{P}<0.05, \mathrm{P}<$ 0.01 and $\mathrm{P}<0.001$, respectively $(t$-test). 


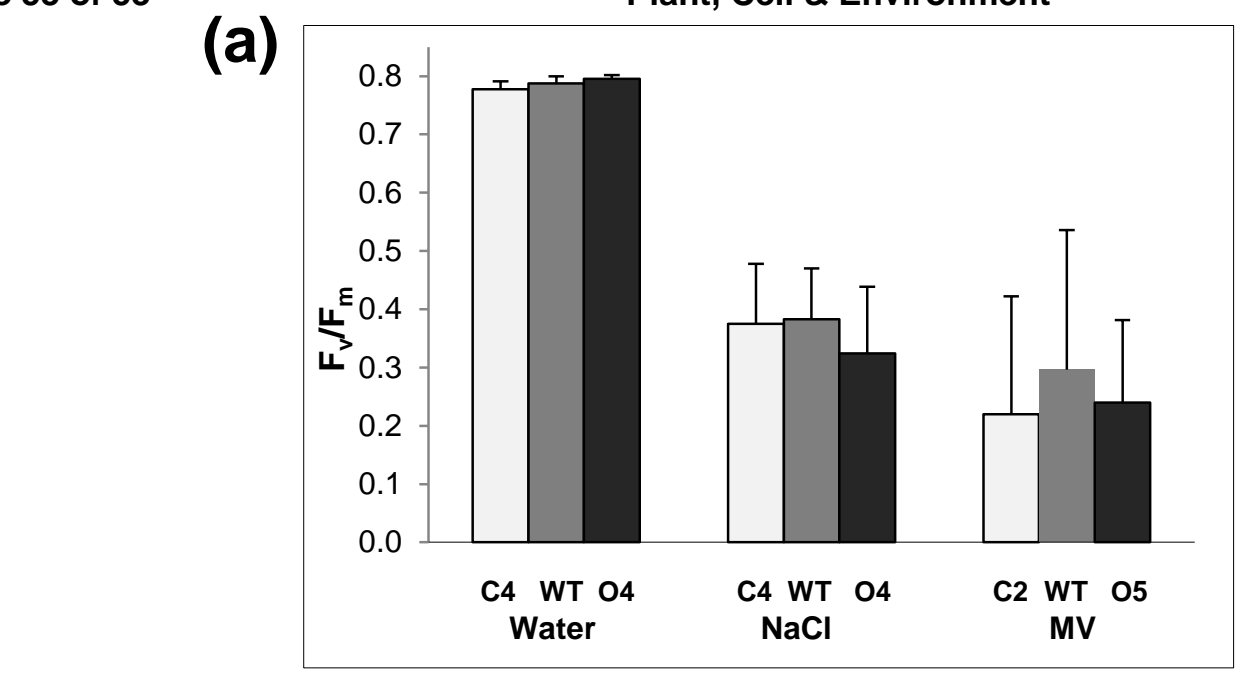

\section{$\begin{array}{llll}\text { (b) } \mathrm{C}-\mathrm{S} 16 \mathrm{C} 2 & \mathrm{C}-\mathrm{S} 16 \mathrm{C} 4 & \mathrm{WT} & \mathrm{OE}-\mathrm{S} 16 \mathrm{O}\end{array}$}

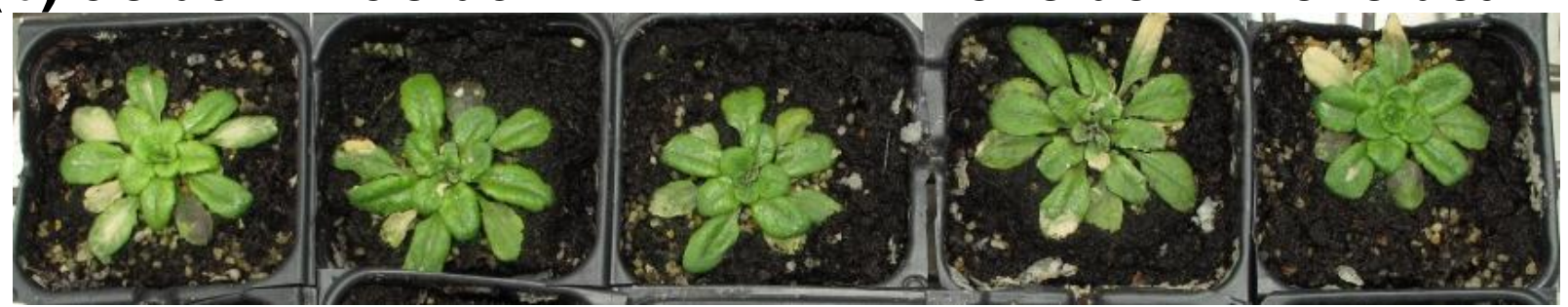

(c)

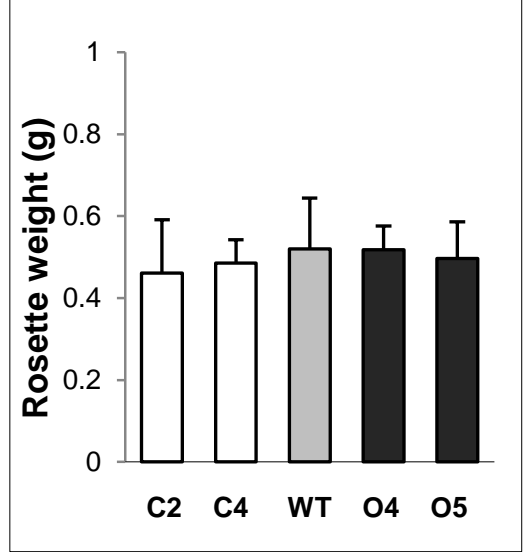

(d)

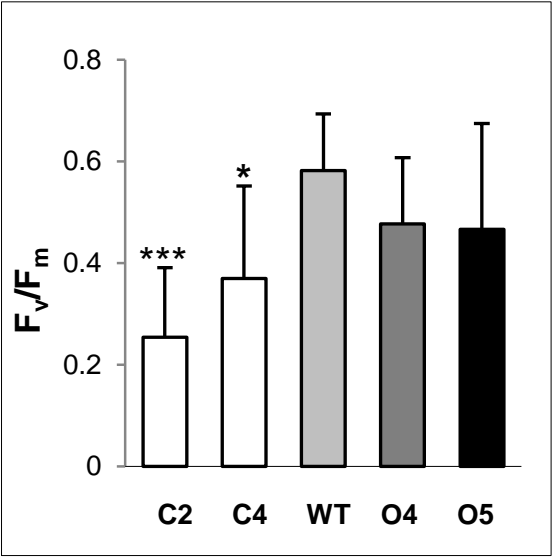

(e)

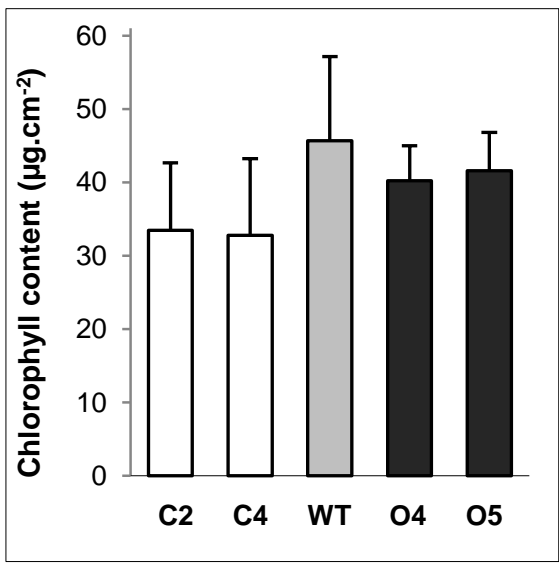

Figure S6. Responses of Arabidopsis plants modified in the expression of GRXS16 to osmotic and oxidative constraints.

(a) Effect of $\mathrm{NaCl}$ and methyl viologen (MV) on the PSII activity of leaf discs. The maximal photochemical efficiency of photosystem II $\left(\mathrm{F}_{\mathrm{v}} / \mathrm{F}_{\mathrm{m}}\right)$ was measured on disks incubated on water, $0.15 \mathrm{M} \mathrm{NaCl}$ or $2 \mu \mathrm{M} \mathrm{MV}$ in the phytotron conditions $\left(200 \mu \mathrm{mol}\right.$ photons. $\mathrm{m}^{-2} . \mathrm{s}^{-1} ; 8$-h photoperiod; $\left.22^{\circ} \mathrm{C}\right)$ for $48 \mathrm{~h}$. Mean $\pm \mathrm{SD}$ of 10 independent measurements. (b-e) Growth and photosynthetic parameters of plants exposed to $\mathrm{NaCl}$. Plants aged of 21 days grown in control conditions were then watered with $0.15 \mathrm{M} \mathrm{NaCl}$ in the phytotron conditions for 31 days (b) Rosette weight of salt-treated plants. Mean \pm SD of 5 independent values. (c) Maximal photochemical efficiency of photosystem II $\left(\mathrm{F}_{\mathrm{v}} / \mathrm{F}_{\mathrm{m}}\right)$ of salt-treated plants. Mean \pm SD of 20 independent measurements performed on 4 plants. (d) Leaf chlorophyll content of salt-treated plants. Mean \pm SD of 5 independent measurements. C-S16 C2 and $\mathrm{C} 4$, lines co-suppressed for GRXS16 expression; WT, wild type; OE-S16 O4 and O5, lines overexpressing GRXS16. * and ***, significantly different from the WT value with $\mathrm{P}<0.05$ and $<0.001$, respectively $(t$-test). 


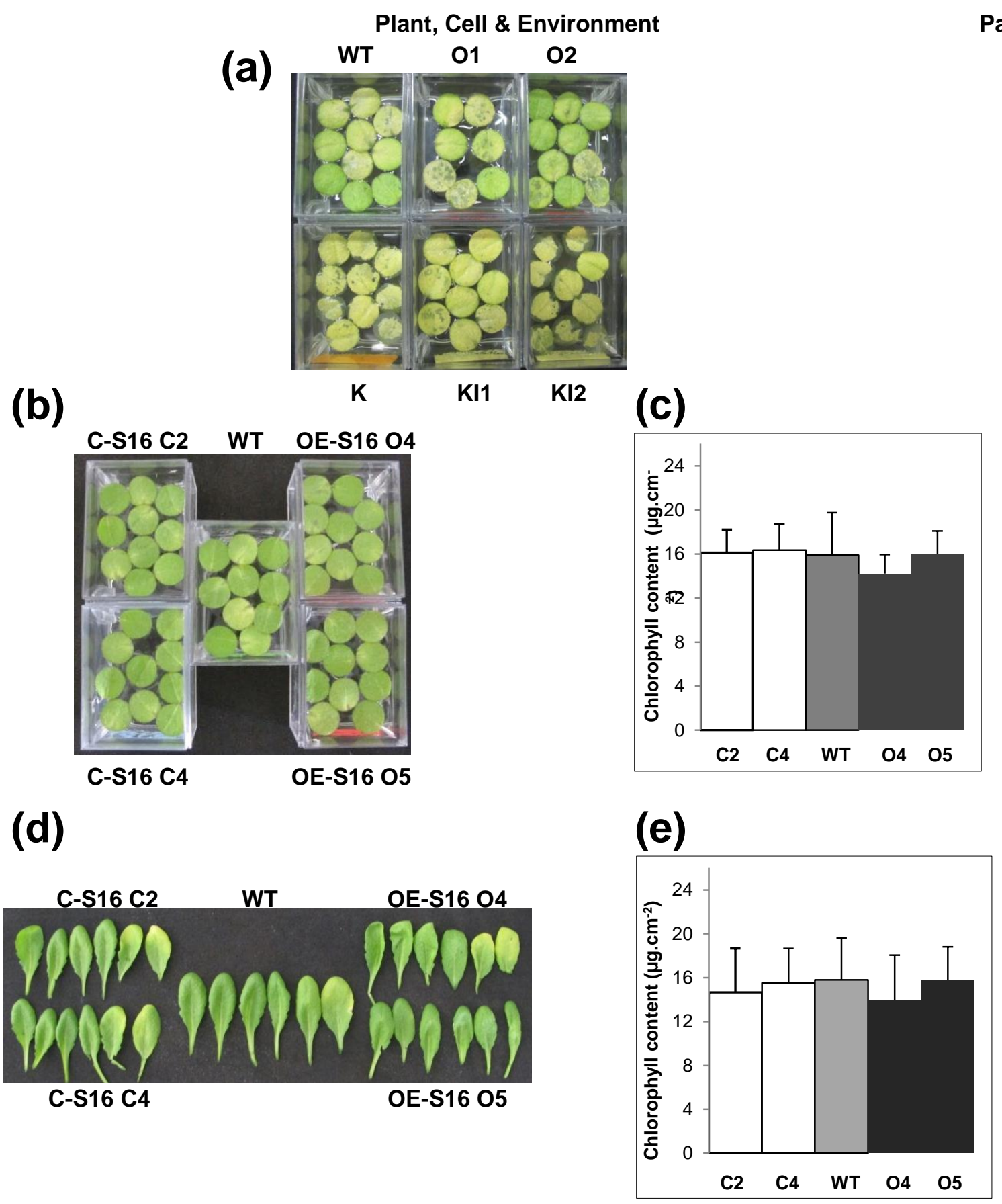

Figure S7. Effect of exposure to dark on the chlorophyll content of Arabidopsis plants modified for GRXS14 and/or GRXS16 expression.

(a, b) Leaf disks incubated on water in the dark for 4 days. (c) Chlorophyll content of disks incubated in the dark for 4 days. Means \pm SD of 6 independent values per genotype. (d) Leaves covered with aluminum foil for 8 days on entire 6-week old plants grown on soil in standard light conditions (8-h photoperiod, $200 \mu$ moles photons. $\mathrm{m}^{-2} \cdot \mathrm{s}^{-1} ; 22^{\circ} \mathrm{C}$ ). (e) Chlorophyll content in leaves covered with aluminum foil for 8 days. Mean \pm SD of 6 independent measurements per genotype. KI2, KI1: lines KO for GRXS14 and RNAi for GRXS16; K, grxS14; WT, wild type; OE-S14 O1 and O2, lines overexpressing GRXS14; C-S16 C2 and C4, lines co-suppressed for GRXS16 expression; OE-S16 O4 and O5, lines overexpressing GRXS16. 